\title{
HÁBITOS ALIMENTARES E OBSTIPAÇÃO \\ INTESTINAL: UMA REVISÃO SISTEMÁTICA
}

\author{
FOOD HABITS AND INTESTINAL CONSTIPATION: A SYSTEMATIC \\ REVIEW \\ HÁBITOS ALIMENTARIOS Y ESTREÑIMIENTO INTESTINAL: UNA \\ REVISIÓN SISTEMÁTICA
}

\author{
Márcia Alexandra Louro Martins (a30319@alunos.ipb.pt)* \\ António José Gonçalves Fernandes (toze@ipb.pt)** \\ Juliana Almeida-de-Souza (julianalmeida@ipb.pt)***
}

\section{RESUMO}

A obstipação intestinal é um problema de saúde que pode ser definido como dificuldade em defecar. e que pode ser tratado com recurso à alimentação. Por isso, o objetivo do estudo é verificar a associação entre o consumo alimentar e a obstipação intestinal. Para tal, foi feita uma pesquisa nas bases de dados PubMed e Web of Science com as palavras-chave diet e constipation. Dos 3857 artigos encontrados, foram selecionados 20 artigos publicados no período de 2007-2017 que respeitavam os critérios de inclusão. Os resultados revelaram que a alimentação está associada à obstipação intestinal. A fibra tem um papel muito importante para a diminuição da obstipação intestinal. Nutrientes como o magnésio e zinco também demonstraram diminuir a obstipação. Alimentos como hortícolas, fruta, batata, ovos, soja, grãos integrais, probióticos, kefir e ingestão de líquidos ajudam a combater a obstipação. Pelo contrário, leite de vaca, iogurte, pão, massa, arroz, bolachas e o padrão alimentar fast-food tendem a aumentar a obstipação intestinal, podendo ser o motivo do aparecimento desta patologia. Conclui-se que os hábitos alimentares estão associados à presença/ausência de obstipação intestinal, sendo importante adotar medidas corretivas para minimizar ou eliminar esta patologia, tendo em atenção os alimentos que se devem ingerir.

Palavras Chave: obstipação intestinal, critérios e escalas, dieta.

\section{ABSTRACT}

Constipation is a health problem that can be defined as difficulty in defecating and that can be addressed by changing eating habits. So, the objective of this study is to verify the association between food consumption and intestinal constipation. For this, a search was conducted in PubMed and Web of Science databases with the keywords diet and constipation. From 3,857 articles found, 20 published in the 2007-2017 period, meeting the inclusion criteria, were selected. Results revealed that food intake is associated with intestinal constipation. Fibre plays a very important role in reducing intestinal constipation. Nutrients such as magnesium and zinc have also been shown to decrease constipation. Foods such as vegetables, fruit, potatoes, eggs, soybeans, whole grains, kefir, probiotics and fluid intake help to fight constipation. On the other hand, cows' milk, yoghurt, bread, pasta, rice, crackers, and a fast-food dietary pattern tend to increase intestinal constipation and may be the reason for the onset of this pathology. It is concluded that eating habits are associated with 
the presence/absence of intestinal constipation, and it is important to adopt corrective measures to minimize or eliminate this pathology, taking into account the foods to be eaten.

Keywords: constipation, criteria and scales, diet.

\section{RESUMEN}

El estreñimiento intestinal es un problema de salud que puede ser definido como dificultad en defecar y puede ser tratada recurriendo a la alimentación. Por eso, el objetivo del estudio es verificar la asociación entre el consumo alimentario y el estreñimiento intestinal. Para ello, se realizó una pesquisa en las bases de datos PubMed y Web of Science con las palabras clave diet y constipation. De los 3857 artículos encontrados, fueron seleccionados 20 artículos publicados en el período 20072017 que respectaban los criterios de inclusión. Se verificó que la alimentación está asociada al estreñimiento intestinal. La fibra tiene un papel muy importante para la disminución del estreñimiento intestinal. Los nutrientes como el magnesio y el cinc, también, han demostrado disminuir el estreñimiento intestinal. Los alimentos como hortalizas, frutas, patatas, huevos, soja, granos integrales, probióticos, kéfir y la ingestión de líquidos ayudan a combatir el estreñimiento. Al contrario, leche de vaca, yogurt, pan, pasta, arroz, galletas, y el patrón alimentario de comida rápida tienden a aumentar el estreñimiento intestinal, pudiendo ser el motivo de la aparición de esta patología. Se concluye que los hábitos alimentarios están asociados a la presencia/ausencia de estreñimiento intestinal, siendo importante adoptar medidas correctivas para minimizar o eliminar esta patología, teniendo en cuenta los alimentos que se deben ingerir.

Palabras clave: estreñimiento intestinal, criterios y escalas, dieta.

*Licenciada em Dietética e Nutrição pela Escola Superior de Saúde do Instituto Politécnico de Bragança.

** Professor Adjunto do Departamento de Ciências Sociais e Exatas, Escola Superior Agrária, Instituto Politécnico de Bragança, Portugal. Mestre em Gestão pela Universidade da Beira Interior, Covilhã, Portugal. Doutorado em Gestão pela Universidade de Trás-os-Montes e Alto Douro, Vila Real, Portugal. Investigador Integrado do Centro de Investigação de Montanha do Instituto Politécnico de Bragança. Campus de Santa Apolónia - 5300-253 Bragança, Portugal.

*** Professora Adjunta do Departamento de Tecnologias de Diagnóstico e Terapêutica, Escola Superior de Saúde, Instituto Politécnico de Bragança, Portugal. Mestre em Saúde Pública pela Universidade do Porto, Portugal. Doutorada em Ciências do Consumo Alimentar e Nutrição pela Faculdade de Ciências da Nutrição e Alimentação da Universidade do Porto, Portugal. Investigadora não integrada Centro de Investigação de Montanha. Campus de Santa Apolónia - 5300-253 Bragança, Portugal.

Submitted: 26 th march 2018

Accepted: 30 th june 2019 


\section{INTRODUÇÃO}

A obstipação intestinal é um problema de saúde que ronda, nas populações da América do Norte entre, os 2\% a $27 \%$ e na Oceânia e Europa 5\% a 35\% (Garcia, Bertolini, Souza, Santos, \& Pereira, 2016). Pode ser definida como dificuldade em defecar. No entanto, há uma ampla diversidade de definições, critérios e escalas publicados.

A obstipação intestinal pode ser classificada como funcional (primária), sendo associada a fatores como sedentarismo, hábitos alimentares e stress, ou orgânica (secundária), pode ser associada a outra doença ou uso de medicação (Gray, 2011).

A obstipação constitui um fator de risco para o cancro do cólon rectal (CCR) uma vez que a presença de uma motilidade intestinal diminuída faz com que o contato de produtos carcinogénicos com a superfície intestinal seja maior e isso possa provocar CCR (Sá, 2008). Também, a qualidade de vida da pessoa fica comprometida pelo impacto de restrições quotidianas e absentismo de atividades sociais (Couto, 2014).

Alguns estudos mostram que o consumo de fibra alimentar e de líquidos podem ser usados na diminuição da obstipação intestinal (Yang, Wang, Zhou, \& Xu, 2012; Stewart \& Schroeder, 2013; Garcia, Bertolini, Souza, Santos, \& Pereira, 2016). Pelo contrário, o leite de vaca está, segundo alguns estudos, na origem da obstipação intestinal (Caffarelli, Baldi, Bendandi, Calzone, Marani, \& Pasquinelli, 2010; Ferreira, Pinto, Carvalho, Gonçalves, Lima, Pereira, 2014). O consumo de kiwi, ameixa e dióspiro diminuem a obstipação e a banana aumenta a obstipação (Bae, 2014).

O uso de próbioticos demonstra, ainda, ser um contrassenso no que diz respeito ao benefício para o tratamento da obstipação intestinal. Efetivamente, estudos referem os benefícios (Flesch, Poziomyck, \& Damin, 2014) e outros afirmam haver poucas evidências (Vandenplas, Huys, \& Daube, 2015) e, ainda, outros que afirmam não ter provocado efeitos eficazes nas crianças (Korterink, Ockeloen, Benninga, Tabbers, Hilbink, \& Deckers-Kocken, 2014).

Como foi referido, há uma ampla diversidade de escalas que podem ser usadas para avaliar a obstipação intestinal, nomeadamente, a escala GSRS (Gastrointestinal Symptom Rating Scale), a escola de Bristol, a escala de Agachan e escala analógica visual (VAS).

A Escala de GSRS é um instrumento específico que tem 15 questões divididas em 5 domínios que cobrem a área gastrointestinal, nomeadamente, diarreia, obstipação, dor abdominal, refluxo e indigestão. As respostas ao questionário são organizadas de acordo com a escala de Likertde 7 pontos, na qual "1" indica ausência e "7" a frequência ou intensidade mais alta dos sintomas (Kulich, Madisch, Pacini, Piqué, Regula, Van Rensburg etal., 2008).

A Escala de Bristol é descritiva e visual e consta de sete tipos de fezes, sendo composta por imagens e respetivas definições como objetivo de avaliar a consistência. Tipo 1: separar grumos duros, como nozes (difícil de passar), tipo 2: em forma de salsicha, mas grumoso, tipo 3: como uma salsicha, mas com rachaduras na sua superfície, tipo 4: como uma salsicha italiana ou cobra, suave e macia, tipo 5: bolhas macias com bordas cortadas (passadas facilmente), tipo 6: peças macias com bordas irregulares e tipo 7: águas, sem peças sólidas (Marques, 2012).

Na Escala de Agachan, a intensidade da obstipação é baseada nas principais queixas relacionadas com as evacuações e características das fezes. Os sintomas incluídos são frequência evacuatória, dificuldade ou esforço para evacuar, dor a evacuar, sensação de evacuação incompleta, dores abdominais, tempo gasto para iniciar a evacuação, tipo de auxílio para evacuação, tentativas falhadas por dia e duração da obstipação. Cada item é classificado 
de 0 a 4. No final, a soma classifica a obstipação, inexistente de 0 a 10, moderada de 11 a 20 e intensa de 21 a 30 (Agachan, Chen, Pfeifer, Reissman, \& Wexner, 1996).

A escala analógica visual - VAS é uma ferramenta de cinco itens projetada para avaliar a frequência e a gravidade dos sintomas de obstipação. Dois dos cinco itens, consistência de fezes e tensão, são classificados em uma escala de Likertque varia de 1 a 5 . A presença de obstipação, duração da obstipação e evacuação incompleta, são classificados numa escala de 0 a 10. Os valores mais próximos de 10 demonstram satisfação intestinal mais elevada (Sharma \& Agarwal, 2012).

Diferentes critérios podem ser usados na definição da obstipação intestinal, nomeadamente, critério de lowa, Roma I, Roma II, Roma III, avaliação da consistência, avaliação do tempo de trânsito colónico (CTT), critérios de NASPGHAN, critério de Boston, entre outros.

No critério de lowa apresenta obstipação quem tem duas ou mais das seguintes características durante as 8 semanas anteriores: menos de três evacuações por semana, um ou mais episódios de incontinência fecal por semana, grandes fezes no reto ou sentida no exame abdominal, fezes grandes que obstruem a sanita, retenção postural e defecação dolorosa (Afzal, Tighe, \& Thomson, 2011). A obstipação foi considerada presente quando o adolescente apresentava evacuações com frequência s três vezes na semana e cuja consistência das fezes foi considerada endurecida ou em cíbalos, além do relato de dificuldade de evacuar sem uso de laxantes (Vitolo, Campagnolo, \& Gama, 2007).

Os critérios de Roma I revelam obstipação quando dois ou mais dos seguintes sintomas surgem pelo menos durante 3 meses: tensão $>25 \%$ do tempo, fezes irregulares/duras $>25 \%$ do tempo, sensação de evacuação incompleta $>25 \%$ do tempo, número de defecações $\leq 2$ por semana, dados insuficientes para o síndrome do intestino irritável (SII). Os critérios não se aplicam quando o paciente toma laxantes (Sanchez \& Bercik, 2011).

Os critérios de Roma II revelam obstipação quando, pelo menos 12 semanas, que não precisam de ser consecutivas, nos 12 meses anteriores à presença de dois ou mais sintomas de: tensão em $>1 / 4$ defecações, fezes húmidas ou duras em defecações $>1 / 4$, sensação de evacuação incompleta em > 1/4 das defecações, sensação de obstrução anorretal/bloqueio em defecações $>1 / 4$, manobras manuais para facilitar > 1/4 defecações (por exemplo, evacuação digital, apoio do períneo) e/ou $\leq 3$ defecações/semana, as fezes soltas não estão presentes e existem critérios de insuficiência para SII (Thompson,. Longstreth, Drossman, Heaton, Irvine, \& Müller-Lissner, 1999).

Os critérios de Roma III revelam obstipação quando se verificam os seguintes critérios gerais: presença durante pelo menos 3 meses durante um período de 6 meses antes do diagnóstico, pelo menos uma de cada quatro evacuações cumpre com critérios específicos, critérios para SII são insuficientes e ausência de fezes ou, raras vezes, fezes de consistência diminuída. Critérios específicos: presença de dois ou mais sintomas. Esforço para evacuar, fezes fragmentadas ou endurecidas, sensação de evacuação incompleta, sensação de obstrução anorretal ou bloqueios, necessidade de manobra manual ou digital para facilitar a evacuação, $<$ de três movimentos intestinais por semana. Roma III em pediatria - presença de $\geq 2$ dos seguintes sintomas, durante 1 mês em crianças com idade $<4$ anos, ou 2 meses em crianças com idade $\geq 4$ anos: $\leq 2$ dejeções por semana, $\geq 1$ episódio por semana de incontinência fecal após a aquisição de controlo de esfíncteres, história de retenção fecal, história de dejeções dolorosas ou duras, elevado volume fecal ao toque rectal, fezes de diâmetro elevado, obstruindo a sanita (Lindberg, Hamid, Malfertheiner, Thomsen, Fernandez, Garisch et al., 2010; Vieira, Negrelle, Webber, Gosdal, Truppel, \& Kusma, 2016). 
Avaliação da consistência (muito firme, firme, normal e macias) e o número de defecações (2 por semana, 3-6 semana e 1 vez por dia (2 ou menos vezes por semana, de 3 a 6 vezes por semana e 1 vez por dia (Salehi, Dehghani, \& Imanieh, 2010).

A avaliação do tempo de trânsito colónico (CTT), a partir da técnica dos marcadores radiopticos. $O$ estudo de retenção de marcadores de 5 dias é um método simples para medir o trânsito colónico que requer a ingestão de marcadores de uma só vez. A realização de uma radiografia simples de abdómen 120 horas após, é suficiente para avaliar o tempo de trânsito colónico através da quantificação dos marcadores presentes. Se persistirem mais de $20 \%$ dos marcadores no cólon, significa que o trânsito é lento. O acúmulo distal dos marcadores pode indicar um transtorno da evacuação e, nos casos típicos de obstipação por trânsito lento, onde quase todos os marcadores se mantêm e se observam tanto no cólon direito como no esquerdo. Um número adequado de marcadores (20-24) podem ser incluídos numa cápsula de gelatina para facilitar a ingestão (Lindberg, Hamid, Malfertheiner, Thomsen, Fernandez, Garisch et al., 2010).

Na guideline de diagnóstico e tratamento da obstipação intestinal na China de 2013, a obstipação foi definida de como a diminuição da frequência de defecação, fezes secas e duras e dificuldade em defecar (Yang, Zhang, Zhu, Tang, Zhao, Li, \& Gabriel, 2016).

Com base nos critérios de NASPGHAN, a definição de obstipação crónica consiste na ocorrência de dois ou mais das seguintes características durante o período anterior de 8 semanas: frequência de evacuações intestinais inferior a três por semana, mais de um episódio de incontinência fecal por semana, a passagem de fezes tão grandes que obstruam a sanita, postura retentora e defecação dolorosa (Benninga, Candy, Catto-Smith, Clayden, LoeningBaucke, Di Lorenzo et al., 2005).

O critério de Boston envolve a presença de dois ou mais sintomas: fezes grandes e duras, defecar menos de 3 vezes por semana, aparecimento de complicações como dor abdominal recorrente, perda de urina, infeções do trato urinário não estrutural e/ou manchas fecais, esta última definida como a passagem involuntária das fezes devido à impacto retal (Hyams, Colletti, Faure, Gabriel-Martinez, Maffei, \& Morais et al., 2002).

CSI (Constipation Severity Instrumenf) compreende 16 perguntas e tem três subescalas. Estas subescalas são a Defecação Obstrutiva (DO), Inércia Colónica $(\mathrm{Cl})$ e Dor. Os scoresmais baixos e maiores foram "0-28 pontos" para OD, "0-29 pontos" para $\mathrm{Cl}$, "0-16 pontos" para dor e "0-73 pontos" para CSI. As pontuações altas mostram que a pessoa tem obstipação intestinal (Arslan \& Hisar, 2016).

Pelo exposto, a obstipação pode ser definida com o hábito intestinal insatisfatório (Machado \& Capelarim, 2010). Neste sentido, o objetivo desta revisão da literatura é verificar se existe associação entre a obstipação intestinal e os hábitos alimentares e, em caso afirmativo, identificar os fatores alimentares que contribuem para aumentar ou diminuir os sintomas associados a esta patologia.

\section{METODOLOGIA}

Para a execução da pesquisa dos artigos foram utilizadas duas bases de dados, designadamente, a Web of Sciencee a PubMed, onde foram utilizadas as palavras-chaves diet e constipation. O processo de seleção dos artigos consistiu em quatro fases, nomeadamente, exclusão dos artigos repetidos; análise do título; análise do resumo; e, leitura integral do artigo considerando os critérios de inclusão e exclusão. Foram incluídos artigos originais e de pesquisa, publicados entre 2007 e 2017 e que procurassem estudar a associação entre a 
alimentação e a obstipação intestinal em amostras de humanos sem outra patologia intestinal, de qualquer idade e sexo.

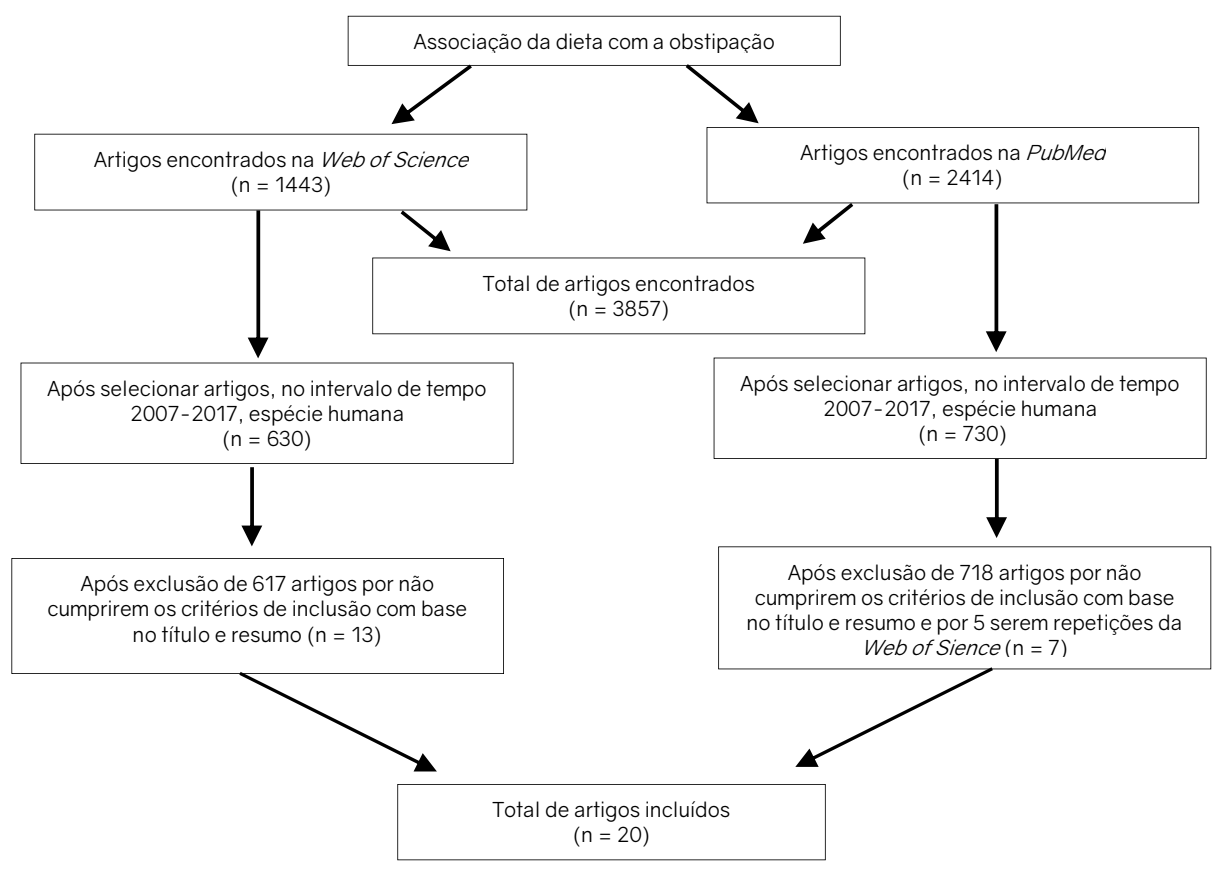

Figura 1 - Diagrama do processo de seleção dos artigos

Os artigos em modelo animal foram excluídos em ambas as bases de dados. Como pode verse na Figura 1, dos 3857 artigos encontrados nas bases de dados consultadas (1443 artigos na Web of Science e 2414 na PubMed), após selecionar artigos, no intervalo de tempo 20072017, espécie humana restaram 630 da Web of Science e 730 da PubMed. Posteriormente, foram excluídos 617 artigos na Web of Science por não cumprirem os critérios de inclusão com base no título e resumo, tendo restado 13 artigos. Na PubMed, após exclusão de 718 artigos por não cumprirem os critérios de inclusão com base no título e resumo e por 5 serem repetições da Web of Sience restaram 7 artigos. Assim, forma incluídos nesta revisão sistemática da literatura 20 artigos a partir dos quais se recolheram os seguintes dados: tipo de estudo, características da amostra, instrumento de recolha de dados, resultados e conclusões, como pode ver-se no Anexo 1.

\section{RESULTADOS}

Dos vinte artigos, oito são experimentais (López Román, Martínez Gonzálvez, Luque, Pons Miñano, Vargas Acosta, Iglesias, \& Hernández, 2008; Irastorza, Ibañez, Delgado-Sanzonetti, Maruri, \& Vitoria, 2010; Machado \& Capelarim, 2010; Salehi, Dehghani, \& Imanieh, 2010; Dehghani, Ahmadpour, Haghighat, Kashef, Imanieh, \& Soleimani, 2012; Turan, Dedeli, Bor, \& Tankut, 2014; Weber, Toporovski, Tahan, Neufeld, \& de Morais, 2014; Kindleysides, KuhnSherlock, Yip, \& Poppitt, 2015), oito transversais (Vitolo, Campagnolo, \& Gama, 2007; Yang, 
Zhang, Zhu, Tang, Zhao, Li, \& Gabriel, 2016; Wu, Pan, Tang, Hwang, Wu, \& Chen, 2011; Tam, Li, \& So, 2012; Asakura, Masayasu, \& Sasaki, 2017; Murakami, Okubo, Takahashi, Hosoi, \& Itabashi, 2007; Chien, Liou, \& Chang, 2011; Inan, Aydiner, Tokuc, Aksu, Ayvaz, Ayhan et al., 2007), dois caso controlo (Lopes \& Victoria, 2008; You, Park, \& Chang, 2010) e dois prospetivos longitudinais (Ayaz \& Hisar, 2014; Maffei \& Vicentini, 2011).

A maioria dos estudos $(n=12)$ foi realizada na Ásia (Salehi, Dehghani, \& Imanieh, 2010; Yang, Zhang, Zhu, Tang, Zhao, Li, \& Gabriel, 2016; Turan, Dedeli, Bor, \& Tankut, 2014; Dehghani, Ahmadpour, Haghighat, Kashef, Imanieh, \& Soleimani, 2012; Wu, Pan, Tang, Hwang, Wu, \& Chen, 2011; Tam, Li, \& So, 2012; Asakura, Masayasu, \& Sasaki, 2017; Murakami, Okubo, Takahashi, Hosoi, \& Itabashi, 2007; Chien, Liou, \& Chang, 2011; Inan, Aydiner, Tokuc, Aksu, Ayvaz, Ayhan et al., 2007; You, Park, \& Chang, J2010; Ayaz, \& Hisar, 2014), cinco na América do Sul (Vitolo, Campagnolo, \& Gama, 2007; Lopes, \& Victoria, 2008; Maffei, \& Vicentini, 2011; Machado, \& Capelarim, 2010; Weber, Toporovski, Tahan, Neufeld, \& de Morais, 2014), dois na Europa (López Román, Martínez Gonzálvez, Luque, Pons Miñano, Vargas Acosta, Iglesias et al., 2008; Irastorza, Ibañez, Delgado-Sanzonetti, Maruri, \& Vitoria, 2010) e um na Oceânia (Kindleysides, Kuhn-Sherlock, Yip, \& Poppitt, 2015).

Em relação às amostras, onze artigos referem-se a estudos em crianças (Vitolo, Campagnolo, \& Gama, 2007; Salehi, Dehghani, \& Imanieh, 2010; Irastorza, Ibañez, Delgado-Sanzonetti, Maruri, \& Vitoria, 2010; Weber, Toporovski, Tahan, Neufeld, \& de Morais, 2014; Dehghani, Ahmadpour, Haghighat, Kashef, Imanieh, \& Soleimani, 2012; Wu, Pan, Tang, Hwang, Wu, \& Chen, 2011; Tam, Li, \& So, 2012; Asakura, Masayasu, \& Sasaki, 2017; Chien, Liou, \& Chang, 2011; Inan, Aydiner, Tokuc, Aksu, Ayvaz, Ayhan et al., 2007; Maffei \& Vicentini, 2011), seis em adultos (Murakami, Okubo, Takahashi, Hosoi, \& Itabashi, 2007; López Román, Martínez Gonzálvez, Luque, Pons Miñano, Vargas Acostalglesias et al., 2008; Lopes \& Victoria, 2008; You, Park, \& Chang, 2010; Ayaz, \& Hisar, 2014; Kindleysides, Kuhn-Sherlock, Yip, \& Poppitt, 2015) e três em amostras que incluíam adultos e idosos (Machado \& Capelarim, 2010; Turan, Dedeli, Bor, \& Tankut, 2014; Yang, Zhang, Zhu, Tang, Zhao, Li, \& Gabriel, 2016).

Um total de oito estudos referem que há uma maior prevalência de obstipação nas mulheres (Lopes \& Victoria, 2008; López Román, Martínez Gonzálvez, Luque, Pons Miñano, Vargas Acosta, Iglesias, \& Hernández, 2008; Salehi, Dehghani, \& Imanieh, 2010; Machado \& Capelarim, 2010; Irastorza, Ibañez, Delgado-Sanzonetti, Maruri, \& Vitoria, 2010; Turan, Dedeli, Bor, \& Tankut, 2014; Weber, Toporovski, Tahan, Neufeld, \& de Morais, 2014; Kindleysides, Kuhn-Sherlock, Yip, \& Poppitt, 2015) e um refere que os homens são mais obstipados (Dehghani, Ahmadpour, Haghighat, Kashef, Imanieh, \& Soleimani, 2012).

Em relação aos instrumentos de recolha de dados (IRD) para avaliar a alimentação, a maioria dos artigos ( $n=8$ ) utilizou o questionário de frequência alimentar (QFA) (Inan, Aydiner, Tokuc, Aksu, Ayvaz, Ayhan et al., 2007; Vitolo, Campagnolo, \& Gama, 2007; Maffei \& Vicentini, 2011; Wu, Pan, Tang, Hwang, Wu, \& Chen, 2011; Tam, Li, \& So, 2012; Ayaz \& Hisar 2014; Yang, Zhang, Zhu, Tang, Zhao, Li, \& Gabriel, 2016; Asakura, Masayasu, \& Sasaki, 2017). Outros estudos reportaram, ainda, a utilização de recordatórios alimentares $(n=3)$ (Vitolo, Campagnolo, \& Gama, 2007; You, Park, \& Chang, 2010; Chien, Liou, \& Chang, 2011), registos alimentares ( $n=3)$ (Lopes \& Victoria, 2008; Irastorza, Ibañez, Delgado-Sanzonetti, Maruri, \& Vitoria, 2010; Dehghani, Ahmadpour, Haghighat, Kashef, Imanieh, \& Soleimani, 2012), sobras de alimentos ou suplementos alimentares fornecidos $(n=2)$ (Weber, Toporovski, Tahan, Neufeld, \& de Morais, 2014; Kindleysides, Kuhn-Sherlock, Yip, \& Poppitt, 2015), questionário da história alimentar $(n=1)$ (Murakami, Okubo, Takahashi, Hosoi, \& Itabashi, 2007) e questionário de adesão alimentar $(n=1)$ (Machado \& Capelarim, 2010). 
No que diz respeito à avaliação da obstipação intestinal, doze estudos basearam-se em questionários (Inan, Aydiner, Tokuc, Aksu, Ayvaz, Ayhan et al., 2007; Lopes \& Victoria, 2008; Murakami, Okubo, Takahashi, Hosoi, \& Itabashi, 2007; You, Park, \& Chang, 2010; Chien, Liou, \& Chang, 2011; Maffei \& Vicentini, 2011; Wu, Pan, Tang, Hwang, Wu, \& Chen, 2011; Tam, Li, \& So, 2012; Ayaz \& Hisar, 2014; Weber, Toporovski, Tahan, Neufeld, \& de Morais, 2014; Yang, Zhang, Zhu, Tang, Zhao, Li, \& Gabriel, 2016; Asakura, Masayasu, \& Sasaki, 2017), cinco estudos em diário fecal (López Román, Martínez Gonzálvez, Luque, Pons Miñano, Vargas Acosta, Iglesias, \& Hernández, 2008; Irastorza, Ibañez, Delgado-Sanzonetti, Maruri, \& Vitoria, 2010; Dehghani, Ahmadpour, Haghighat, Kashef, Imanieh, \& Soleimani, 2012; Turan, Dedeli, Bor, \& Tankut, 2014; Kindleysides, Kuhn-Sherlock, Yip, \& Poppitt, 2015) e dois foram obtidos por entrevista (Vitolo, Campagnolo, \& Gama, 2007; Machado \& Capelarim, 2010).

Os critérios de definição da obstipação mais utilizados foram os de Roma III em seis estudos (Irastorza, Ibañez, Delgado-Sanzonetti, Maruri, \& Vitoria, 2010; Dehghani, Ahmadpour, Haghighat, Kashef, Imanieh, \& Soleimani, 2012; Weber, Toporovski, Tahan, Neufeld, \& de Morais, 2014; Tam, Li, \& So, 2012; Kindleysides, Kuhn-Sherlock, Yip, \& Poppitt, 2015; Asakura, Masayasu, \& Sasaki, 2017) seguido de Roma II com cinco estudos (López Román, Martínez Gonzálvez, Luque, Pons Miñano, Vargas Acosta, Iglesias, \& Hernández, 2008; Lopes \& Victoria, 2008; Machado \& Capelarim, 2010; You, Park, \& Chang, 2010; Turan, Dedeli, Bor, \& Tankut, 2014) e, finalmente, os critérios de Roma I com apenas um estudo (Murakami, Okubo, Takahashi, Hosoi, \& Itabashi, 2007).

Os resultados de oito artigos demonstraram que o consumo de fibra alimentar tem um papel importante na diminuição da obstipação intestinal (Vitolo, Campagnolo, \& Gama, 2007; López Román, Martínez Gonzálvez, Luque, Pons Miñano, Vargas Acosta, Iglesias, \& Hernández, 2008; Machado \& Capelarim, 2010; Maffei \& Vicentini, 2011; Tam, Li, \& So, 2012; Ayaz \& Hisar, 2014; Weber, Toporovski, Tahan, Neufeld, \& de Morais, 2014; Yang, Zhang, Zhu, Tang, Zhao, $\mathrm{Li}, \&$ Gabriel, 2016), enquanto que três estudos não encontraram uma associação entre a fibra alimentar e a obstipação intestinal (Murakami, Okubo, Takahashi, Hosoi, \& Itabashi, 2007; Lopes \& Victoria, 2008, You, Park\& Chang, 2010).

Em relação a outros nutrientes, pouco foi publicado, mas há alguns estudos que afirmam que o consumo de hidratos de carbono (You, Park, \& Chang, 2010; Asakura, Masayasu, \& Sasaki, 2017), zinco e magnésio (Asakura, Masayasu, \& Sasaki, 2017), funcionam como fatores que diminuem a obstipação intestinal. Murakami, Okubo, Takahashi, Hosoi e Itabashi (2007) afirmam não haver relação entre o consumo de magnésio e a obstipação. O consumo de lípidos diminui, igualmente, a obstipação intestinal (Asakura, Masayasu, \& Sasaki, 2017). Já, You, Park e Chang (2010) afirmam que o consumo de lípidos de origem vegetal está associado à presença da obstipação intestinal. Os mesmos investigadores não encontraram relação entre as vitaminas $A, B, C$, o colesterol e o ácido fólico e a obstipação intestinal. Por seu lado, no mesmo estudo, o cálcio animal e vegetal demonstraram aumentar a obstipação. Já, segundo Asakura, Masayasu e Sasaki (2017), a proteína total diminui a obstipação e segundo You, Park e Chang (2010), a proteína vegetal aumenta a obstipação. No estudo de You, Park e Chang (2010), a taurina (aminoácido) não demonstrou ter qualquer relação com a obstipação.

Chien, Liou e Chang (2011) referem que o consumo de líquidos diminui a obstipação. Salehi, Dehghani, \& Imanieh (2010) e Ayaz \& Hisar (2014) especificam que a água diminui a obstipação, Murakami, Okubo, Takahashi, Hosoi, \& Itabashi (2007) relatam que a água nos alimentos diminui a obstipação e, por fim, Lindberg, Hamid, Malfertheiner, Thomsen, Fernandez, Garisch et al. (2010) referem que o sumo de fruta aumenta a obstipação intestinal.

Relativamente a alimentos e grupos de alimentos, Inan, Aydiner, Tokuc, Aksu, Ayvaz, Ayhan et al. (2007), Salehi, Dehghani e Imanieh (2010), Chien, Liou e Chang (2011), Wu, Pan, Tang, 
Hwang, Wu e Chen (2011), Ayaz e Hisar (2014), Yang, Zhang, Zhu, Tang, Zhao, Li e Gabriel (2016) e Asakura, Masayasu e Sasaki (2017) referem que as hortícolas e as frutas diminuem a obstipação enquanto que Chien, Liou e Chang (2011) não encontraram relação entre o consumo de extrato de fruta e a obstipação intestinal.

Em relação às leguminosas, Asakura, Masayasu e Sasaki (2017) mostraram que estas contribuem para a diminuição da obstipação intestinal. Os mesmos investigadores demonstraram que o consumo de carne diminui a obstipação. No mesmo estudo, o peixe não mostrou estar relacionado com a obstipação.

Relativamente aos laticínios, mais especificamente, em relação ao leite, existem três estudos que afirmam que o leite aumenta a obstipação (Irastorza, Ibañez, Delgado-Sanzonetti, Maruri, \& Vitoria, 2010; Salehi, Dehghani, \& Imanieh, 2010; Dehghani, Ahmadpour, Haghighat, Kashef, Imanieh, \& Soleimani, 2012) e, relativamente ao iogurte, Salehi, Dehghani e Imanieh (2010) afirmam que é obstipante. No entanto, o estudo de Turan, Dedeli, Bor e Tankut (2014) sobre o kefir indicou que este diminui a obstipação.

Outros alimentos ou grupos de alimentos foram pouco estudados, mas Chien, Liou e Chang (2011) mostraram que o consumo de grãos integrais contribuem para a diminuição da obstipação. Em particular, Asakura, Masayasu e Sasaki (2017) referem o arroz como fator que aumenta a obstipação intestinal enquanto Inan, Aydiner, Tokuc, Aksu, Ayvaz, Ayhan et al. (2007) não encontraram a mesma relação. O ovo, a soja são referidos por Wu, Pan, Tang, Hwang, Wu e Chen (2011) e a batata por Asakura, Masayasu e Sasaki (2017) como alimentos que contribuem para a diminuição da obstipação. Inan, Aydiner, Tokuc, Aksu, Ayvaz, Ayhan et al. (2007) referem que o queijo, a massa, o pão e as bolachas aumentam a obstipação intestinal. Contudo, Asakura, Masayasu e Sasaki (2017) referem que o pão não tem qualquer relação com a obstipação. Comida gordurosa e gelados não mostraram estar relacionados com a obstipação intestinal (Wu, Pan, Tang, Hwang, Wu, \& Chen, 2011). O único padrão alimentar encontrado nesta revisão foi o consumo de alimentos fast food como influenciador da obstipação intestinal (Tam, Li, \& So, 2012).

\section{DISCUSSÃO E CONCLUSÃO}

A obstipação pode estar presente em qualquer faixa etária. No entanto, a amioria dos estudos foram realizados em grupos com crianças (Vitolo, Campagnolo, \& Gama, 2007; Salehi, Dehghani, \& Imanieh, 2010; Irastorza, Ibañez, Delgado-Sanzonetti, Maruri, \& Vitoria, 2010; Weber, Toporovski, Tahan, Neufeld, \& de Morais, 2014; Dehghani, Ahmadpour, Haghighat, Kashef, Imanieh, \& Soleimani, 2012; Wu, Pan, Tang, Hwang, Wu, \& Chen, 2011; Tam, Li, \& So, 2012; Asakura, Masayasu, \& Sasaki, 2017; Chien, Liou, \& Chang, 2011; Inan, Aydiner, Tokuc, Aksu, Ayvaz, Ayhan et al., 2007; Maffei \& Vicentini, 2011). A literatura defende que a obstipação é mais frequente quanto maior a idade devido às alterações fisiológicas no envelhecimento, às condições de saúde, ao uso de medicação e mobilidade reduzida que predispõem o aparecimento de obstipação intestinal. Apesar disso, apenas três estudos (Machado, \& Capelarim, 2010; Turan, Dedeli, Bor, \& Tankut, 2014; Yang, Zhang, Zhu, Tang, Zhao, Li, \& Gabriel, 2016; Garcia, Bertolini, Souza, Santos, \& Pereira, 2016) afirmam, diretamente, envolver idosos.

No presente estudo, em geral, a obstipação intestinal é predominante em grupos do sexo feminino (Lopes \& Victoria, 2008; López Román, Martínez Gonzálvez, Luque, Pons Miñano, Vargas Acosta, Iglesias, \& Hernández, 2008; Salehi, Dehghani, \& Imanieh, 2010; Machado \& Capelarim, 2010; Irastorza, Ibañez, Delgado-Sanzonetti, Maruri, \& Vitoria, 2010; Dehghani, 
Ahmadpour, Haghighat, Kashef, Imanieh, \& Soleimani, 2012; Turan, Dedeli, Bor, \& Tankut, 2014; Weber, Toporovski, Tahan, Neufeld, \& de Morais, 2014; Kindleysides, Kuhn-Sherlock, Yip, \& Poppitt, 2015). Tal facto pode ser explicado por alterações hormonais, assim como fatores comportamentais, histórias de abuso sexual, físico e emocional e danos na musculatura do períneo durante cirurgias ginecológicas e obstétricas (Garcia, Bertolini, Souza, Santos, \& Pereira, 2016).

O QFA foi o IRD mais utilizado (Inan, Aydiner, Tokuc, Aksu, Ayvaz, Ayhan et al., 2007; Vitolo, Campagnolo, \& Gama, 2007; Maffei, \& Vicentini, 2011; Wu, Pan, Tang, Hwang, Wu, \& Chen, 2011; Tam, Li, \& So, 2012; Ayaz \& Hisar 2014; Yang, Zhang, Zhu, Tang, Zhao, Li, \& Gabriel, 2016; Asakura, Masayasu, \& Sasaki, 2017) que estima a ingestão habitual do indivíduo, não altera o padrão de consumo e é de baixo custo. No entanto, depende da memória dos hábitos alimentares passados e de habilidades cognitivas para estimar o consumo médio num longo período de tempo. Razão pela qual, em amostras de indivíduos com idade mais avançada, pode haver dificuldades pois o desenho do instrumento requer esforço e tempo. Para além disso, pode haver dificuldades na sua aplicação conforme o número e a complexidade da lista de alimentos. Outra dificuldade na sua aplicação pode surgir em estudos com crianças pois, mesmo que elas já saibam escrever, é necessário haver a ajuda de uma pessoa adulta, por ser um tipo de instrumento complexo. A quantificação resultante deste instrumento revela ser pouco exata pois estima o consumo absoluto. Uma vez que nem todos os alimentos consumidos pelo indivíduo podem constar na lista, pode ocorrer algum viés de informação. Portanto, ao definir-se o IRD é importante analisar o estudo como um todo e verificar que tipo de grupos se pretende estudar e que desvantagens podem ocorrer (Andre, Rodriguez, \& Moraes-Filho, 2000).

Em relação à obstipação, a recolha de dados a partir de questionários predominou (Inan, Aydiner, Tokuc, Aksu, Ayvaz, Ayhan et al., 2007; Lopes \& Victoria, 2008; Murakami, Okubo, Takahashi, Hosoi, \& Itabashi, 2007; You, Park, \& Chang, 2010; Chien, Liou, \& Chang, 2011; Maffei \& Vicentini, 2011; Wu, Pan, Tang, Hwang, Wu, \& Chen, 2011; Tam, Li, \& So, 2012; Ayaz \& Hisar, 2014; Weber, Toporovski, Tahan, Neufeld, \& de Morais, 2014; Yang, Zhang, Zhu, Tang, Zhao, Li, \& Gabriel, 2016; Asakura, Masayasu, \& Sasaki, 2017). Como foi referido, muitos estudos foram realizados em crianças o que, certamente, implicou que alguém que saiba ler e escrever tenha feito o preenchimento do questionário. Também, os critérios de Roma prevaleceram como forma de definição da obstipação. Tal facto, implica que o inquirido consiga recordar sintomas. Como foi referido, nos critérios de Roma I, para haver obstipação tem de se verificar dois ou mais sintomas nos últimos 3 meses. Nos critérios de Roma II, tem de haver sintomas nos últimos 12 meses, durante 12 semanas não sendo preciso serem consecutivas e, nos critérios de Roma III, durante três meses embora os sintomas possam ter tido início 6 meses antes do diagnóstico, podendo criar viés de memória e de informação (Sanchez \& Bercik, 2011).

A fibra alimentar mostrou ter um papel importante na diminuição da obstipação (Vitolo, Campagnolo, \& Gama, 2007; López Román, Martínez Gonzálvez, Luque, Pons Miñano, Vargas Acosta, Iglesias, \& Hernández, 2008; Machado \& Capelarim, 2010; Maffei \& Vicentini, 2011; Tam, Li, \& So, 2012; Ayaz \& Hisar, 2014; Weber, Toporovski, Tahan, Neufeld, \& de Morais, 2014; Yang, Zhang, Zhu, Tang, Zhao, Li, \& Gabriel, 2016). Contudo, três estudos demonstram que o consumo de fibra alimentar (Murakami, Okubo, Takahashi, Hosoi, \& Itabashi, 2007; Lopes \& Victoria, 2008) e nutrientes de origem vegetal (You, Park, \& Chang, 2010) não tem qualquer influência na obstipação. Assim sendo, a fibra dietética pode não ser suficiente para tratar pessoas com obstipação intestinal, podendo haver outros fatores biológicos associados.

O facto da obstipação ser predominante com o consumo de hidratos de carbono (You, Park, \& Chang, 2010; Asakura, Masayasu, \& Sasaki, 2017) e comida rápida (Tam, Li, \& So, 2012) pode 
ser explicado pelo facto destes alimentos serem pobres em fibra (Garcia, Bertolini, Souza, Santos, \& Pereira, 2016). Tal explicação, também, pode ser aplicada ao arroz, massa, pão e bolachas (Inan, Aydiner, Tokuc, Aksu, Ayvaz, Ayhan et al., 2007). O consumo de líquidos é importante dado o seu poder de aumentar o número de reflexos gastrocólicos e a sua contribuição para a lubrificação intestinal (Garcia, Bertolini, Souza, Santos, \& Pereira, 2016). De facto, um estudo considera que o consumo de líquidos diminuiu a obstipação (Chien, Liou, \& Chang, 2011), dois especificam que a água diminui a obstipação (Salehi, Dehghani, \& Imanieh, 2010; Ayaz \& Hisar, 2014), outro relata que a água nos alimentos diminui a obstipação (Murakami, Okubo, Takahashi, Hosoi, \& Itabashi, 2007) e, por fim, um refere que o sumo de fruta aumenta a obstipação intestina (Salehi, Dehghani, \& Imanieh, 2010).

Em relação à gordura, em geral, Asakura, Masayasu e Sasaki (2017) referem que a gordura diminui a obstipação e You, Park e Chang (2010) referem que a gordura vegetal aumenta a obstipação. Não foi possível esclarecer estes resultados contraditórios pois não se encontrou literatura capaz de explicar este facto. Apenas se conseguiu encontrar um estudo que referia que a gordura saturada pode aumentar a obstipação, havendo, no entanto, poucas evidências científicas (Taba Taba Vakili, Nezami, Shetty, Chetty, \& Srinivasan, 2015).

Importante, também, é o consumo de frutas e hortícolas (Inan, Aydiner, Tokuc, Aksu, Ayvaz, Ayhan et al., 2007; Salehi, Dehghani, \& Imanieh, 2010; Chien, Liou, \& Chang, 2011; Wu, Pan, Tang, Hwang, Wu, \& Chen, 2011; Ayaz \& Hisar, 2014; Yang, Zhang, Zhu, Tang, Zhao, Li, \& Gabriel, 2016; Asakura, Masayasu, \& Sasaki, 2017) por estarem associados à minimização da obstipação intestinal, havendo apenas um artigo onde o extrato de kiwi não tinha qualquer relação com a obstipação e o sumo de fruta aumentava a obstipação intestinal. Tal facto poderá ser explicado por haver perda de fibra quando processados (Guerra, Rêgo, Silva, Ferreira, Mansilha, Antunes, \& Ferreira, 2012). Contudo, em relação ao consumo de magnésio, é importante a realização de mais estudos uma vez que só foi encontrado um estudo que estabelecia relação com a obstipação, sendo importante de referir que frutas e legumes são ricas em magnésio (Asakura, Masayasu, \& Sasaki, 2017).

Asakura, Masayasu e Sasaki (2017) confirmam que as leguminosas diminuem a obstipação. No entanto, Bernaud e Rodrigues (2013) afirmam que a proteína vegetal aumenta a obstipação, acabando por gerar alguma controvérsia uma vez que as leguminosas são ricas em proteína vegetal.

Os grãos integrais (Chien, Liou, \& Chang, 2011) e a batata (Asakura, Masayasu, \& Sasaki, 2017), também, demonstraram ser laxantes, possivelmente, segundo Bernaud e Rodrigues (2013), devido ao conteúdo em fibra que esses alimentos têm.

O ovo, por sua vez, demonstrou ajudar na diminuição da obstipação. No entanto, é pobre em fibra, não tendo sido encontrada uma possível explicação para esse facto (Bernaud \& Rodrigues, 2013).

Apesar do uso de próbioticos (são microrganismos vivos que quando administrados em quantidades adequadas conferem um benefício para a saúde no hospedeiro), se mostrar benéfico para evitar a obstipação (Flesch, Poziomyck, \& Damin, 2014; Turan, Dedeli, Bor, \& Tankut, 2014), ainda há poucas evidências sobre esse facto (Vandenplas, Huys, \& Daube, 2015), sendo importante a realização de mais estudos.

O consumo de iogurte demonstrou aumentar a obstipação (Inan, Aydiner, Tokuc, Aksu, Ayvaz, Ayhan et al., 2007; Salehi, Dehghani, \& Imanieh, 2010). Tal facto poderá ser explicado pela quantidade de probiótico ser, ainda, insuficiente No entanto, é importante a realização de mais estudos, também, para tornar esta justificação aceitável.

Já, em relação ao consumo de leite de vaca, demonstrou-se que aumenta a obstipação intestinal (López Román, Martínez Gonzálvez, Luque, Pons Miñano, Vargas Acosta, Iglesias, \& 
Hernández, 2008; Irastorza, Ibañez, Delgado-Sanzonetti, Maruri, \& Vitoria, 2010; Salehi, Dehghani, \& Imanieh, 2010). Tal situação pode estar associada à alergia da proteína do leite de vaca, sendo necessário adaptar a alimentação à alergia presente como forma de minimizar/tratar a patologia (Ferreira, Pinto, Carvalho, Gonçalves, Lima, \& Pereira, 2014).

Conclui-se que a fibra alimentar desempenha um papel importante como tratamento preventivo da obstipação intestinal. Alimentos como frutas e hortícolas demonstraram, também, desempenhar um papel importante na diminuição da obstipação intestinal, ao contrário do leite. Contudo, ainda é preciso fazerem-se mais estudos devido aos resultados contraditórios encontrados na literatura e à falta de informação.

\section{Agradecimentos}

Os autores agradecem à Fundação para a Ciência e a Tecnologia (FCT, Portugal) e ao FEDER no âmbito do programa PT2020 pelo apoio financeiro ao CIMO (UID/AGR/00690/2013).

\section{BIBLIOGRAFIA}

Afzal, N., Tighe, M. e Thomson, M. (2011). Constipation in children Ital J Pediatr. 37: 28.

Agachan, F., Chen, T., Pfeifer, J., Reissman, P. \& Wexner, S. (1996). A Constipation Scoring System to simplify Evaluation and Management of Constipated Patients. Dis Colon Rectum. 39 (6): 681-685.

Andre, S., Rodriguez, T. \& Moraes-Filho, J. (2000). Constipação intestinal. Revista Brasileira de Medicina, São Paulo. 57 (12): 53-63.

Arslan, H. \& Hisar, K. (2016). Determination of the constipation status of the university students staying at girls dormitory. TAF Preventive Medicine Bulletin. 15 (4): 330-335.

Asakura, K., Masayasu, S. \& Sasaki, S. (2017). Dietary intake, physical activity, and time management are associated with constipation in preschool children in japan. Asia Pac J Clin Nutr. 26 (1): 118-129.

Ayaz, S. \& Hisar, F. (2014). The efficacy of education programme for preventing constipation in women. Int J Nurs Pract. 20 (3): 275-282.

Bae, S. (2014). Diets for constipation. Pediatr Gastroenterol Hepatol Nutr. 17 (4): 203-208.

Benninga, M., Candy, D., Catto-Smith, A., Clayden, G., Loening-Baucke, V., Di Lorenzo, C., Nurko, S., \& Staiano, A. (2005). The Paris Consensus on Childhood Constipation Terminology (PACCT) Group. J Pediatr Gastroentero/ Nutr. 40: 273-275.

Bernaud, F. \& Rodrigues, T. (2013). Fibra Alimentar-Ingestão adequada e efeitos sobre a saúde do metabolismo. Arq Bras Endocrino/ Metab. 57 (6): 387-405.

Carlo Caffarelli, C., Baldi, F.,Bendandi, B., Calzone, L., Marani, M. \& Pasquinelli, P. (2010). Cow's milk protein allergy in children: a practical guide. Ital J Pediatr. 36: 5.

Chien, L., Liou, Y. \& Chang, P. (2011). Low defaecation frequency in Taiwanese adolescents: Association with dietary intake, physical activity and sedentary behavior. $J$ Paediatr Child Health. 47 (6): 381-386.

Couto, J. (2014). Obstipação crónica no idoso: opções terapêuticas. Trabalho final do Mestrado integrado em Medicina. Coimbra: Faculdade de Medicina da Universidade de Coimbra.

Dehghani, S.-M., Ahmadpour, B., Haghighat, M., Kashef, S., Imanieh, M., \& Soleimani, M. (2012). The role of cow's milk allergy in pediatric chronic constipation: a randomized clinical trial. Iran J Pediatr. 22 (4): 468-474. 
Ferreira, S., Pinto, M., Carvalho, P., Gonçalves, J., Lima, R. \& Pereira, F., (2014). Alergia às proteínas do leite de vaca com manifestações gastrointestinais. Nascer e crescer - revista de pediatria do centro hospitalar do Porto. 23 (2): 72-79.

Fisberg, R., Marchioni, D. \& Colucci, A. (2009). Avaliação do consumo alimentar e da ingestão de nutrientes na prática clínica. Arq Bras Endocrino/Metab. 53 (5): 617-624.

Flesch, A., Poziomyck, A. \& Damin, D. (2014). O uso terapêutico dos simbióticos. Arq Bras Cir Dig. 27 (3): 206-209.

Garcia, L., Bertolini, S., Souza, M., Santos, M. \& Pereira, C. (2016). Constipação intestinal: aspectos epidemiológicos. Revista Saúde e Pesquisa. 9 (1): 153-162.

Gray, J. (2011).What is chronic constipation? Definition and diagnosis. Can J Gastroenterol. 25 (Suppl B): 7B-10B.

Guerra, A., Rêgo, C., Silva, D., Ferreira, G., Mansilha, H., Antunes, H. \& Ferreira, R. (2012). Alimentação e nutrição do lactente. Acta Pediátrica Port. 43 (2): S17-S40.

Hyams, J., Colletti, R., Faure, C., Gabriel-Martinez, E., Maffei, H., Morais, M., Hock, Q. \& Vandenplas, Y. (2002). Functional gastrointestinal disorders: Working Group Report of the First World Congress of Pediatric Gastroenterology, Hepatology, and Nutrition. J Pediatr Gastroentero/ Nutr. 35 (suppl 2): S110-S117.

Inan, M., Aydiner, C., Tokuc, B., Aksu, B., Ayvaz, S., Ayhan, S., Ceylan, T., \& Basaran, U. (2007). Factors associated with childhood constipation. J Paediatr Child Health. 43: 700-706.

Irastorza, I., Ibañez, B., Delgado-Sanzonetti, L., Maruri, N. \& Vitoria, J. (2010). Cow's-Milk-free Diet as a Therapeutic Option in Childhood Chronic Constipation. Journal of pediatric gastroenterology and nutrition. 51 (2): 171-176

Kindleysides, S., Kuhn-Sherlock, B., Yip, W. \& Poppitt, S. (2015). Encapsulated green kiwifruit extract: a randomised controlled trial investigating alleviation of constipation in otherwise healthy adults. Asia Pac J Clin Nutr. 24 (3): 421-429.

Korterink, J., Ockeloen, L., Benninga, M., Tabbers, M., Hilbink, M. \& Deckers-Kocken, J. (2014). Probiotics for childhood functional gastrointestinal disorders: a systematic review and metaanalysis. Acta Paediatr. 103 (4): 365-372.

Kulich, K., Madisch, A., Pacini, F., Piqué, J., Regula, J,, Van Rensburg, C., Ujszászy, L., Carlsson, J., Halling, K. \& Wiklund, I. (2008). Reliability and validity of the Gastrointestinal Symptom Rating Scale (GSRS) and Quality of Life in Reflux and Dyspepsia (QOLRAD) questionnaire in dyspepsia: A sixcountry study. Health Qual Life Outcomes. 6: 12.

Lindberg, G., Hamid, S., Malfertheiner, P., Thomsen, O., Fernandez, L., Garisch, J., Thomson, A., Goh, K., Tandon, R., Fedail, S., Wong, B., Khan, A., Krabshuis, J. \& Le Mair, A. (2010). Constipação: uma prespectiva mundial. Milwaukee: World Gastroenterology Organisation,

Lopes, A. \& Victoria, C. (2008). Ingestão de fibra alimentar e tempo de trânsito colônico em pacientes com constipação funcional. Arq. Gastroenterol. 45 (1): 58-63.

López Román, J., Martínez Gonzálvez, A., Luque, A., Pons Miñano, J., Vargas Acosta, A., Iglesias, J. \& Hernández, M. (2008). Efecto de la ingesta de un preparado lácteo con fibra dietética sobre el estreñimiento crónico primario idiopático. Nutrición Hospitalaria, 23 (1): 12-19.

Machado, W. \& Capelarim, S. (2010). Avaliação da eficácia e do grau de adesão ao uso prolongado de fibra dietética no ao uso prolongado de fibra dietética. Revista de Nutrição, 23 (2): 231-238.

Maffei, H. \& Vicentini, A. (2011). Prospective Evaluation of Dietary Treatment in Childhood Constipation: High Dietary Fiber and Wheat Bran Intake Are Associated With Constipation Amelioration. J Pediatr Gastroentero/ Nutr. 52 (1): 55-59.

Marques, A. (2012). Síndrome do Intestino Irritável. Trabalho final do Mestrado integrado em Medicina. Porto: Instituto de Ciência Biomédicas Abel Salazar da Universidade do Porto. 
Murakami, K., Okubo, H., Takahashi, Y., Hosoi, Y. \& Itabashi, M. (2007). Association between dietary fiber, water and magnesium intake and functional constipation. Eur J Clin Nutr. 61 (5): 616-622.

Sá, P. (2008). Cancro do cólon e recto. Tese de Mestrado em Medicina. Covilhã: Faculdade de Ciências da Saúde da Universidade da Beira Interior.

Salehi, M., Dehghani, M. \& Imanieh, M. (2010). Therapy of chronic functional constipation in children before and after dietary education. Iranian Red Crescent Medical Journal. 12 (2): 118-121.

Sanchez, M. \& Bercik, P. (2011). Epidemiology and burden of chronic constipation. Can J Gastroenterol. 25 (Suppl B): 11B-15B.

Sharma, S. \& Agarwal, B. (2012)Scoring Systems in Evaluation of Constipation and Obstructed Defecation Syndrome (ODS). JIMSA. 25 (1): 57-59.

Stewart, M. \& Schroeder, N. (2013). Dietary treatments for childhood constipation: efficacy of dietary fiber and whole grains. Nutrition Reviews. 71 (2): 98-109,

Taba Taba Vakili, S., Nezami, B., Shetty, A., Chetty, V. \& Srinivasan, S. (2015). Association of High Dietary Saturated Fat Intake and Uncontrolled Diabetes with Constipation: Evidence from the National Health and Nutrition Examination Survey (NHANES). Neurogastroenterol Motil. 27 (10): 1389-1397.

Tam, Y., Li, A. \& So, H. (2012). Socioenvironmental Factors Associated With Constipation in Hong Kong Children and Rome III Criteria. Journal of Pediatric Gastroenterology and Nutrition. 55 (1): 56 61.

Thompson, W.,. Longstreth, G., Drossman, D., Heaton, K., Irvine, E., Müller-Lissner, S. (1999). Functional bowel disorders and functional abdominal pain. Gut. 45 (Suppl II): ||43-||47

Turan, I., Dedeli, O., Bor, S. \& Tankut, i. (2014). Effects of a kefir supplement on symptoms, colonic transit, and bowel satisfaction score in patients with chronic constipation: a pilot study. Turk $J$ Gastroenterol. 25, 650-656.

Vandenplas, Y., Huys, G. \& Daube, G. (2015). Probiotics: an update. J Pediatr (Rio J). 91 (1): 6-21.

Vieira, M., Negrelle, I., Webber, K., Gosdal, M., Truppel, S. \& Kusma, S. (2016). Pediatrician's knowledge on the approach of functional constipation Revista Paulista de Pediatria (English Edition). 34 (4): 425-431.

Vitolo, M., Campagnolo, P. \& Gama, C. (2007). Factors associated with risk of low dietary fiber intake in adolescents. Jornal de Pediatria. 83 (1): 47-52.

Weber, T., Toporovski, M., Tahan, S., Neufeld, C. \& de Morais, M.. (2014). Dietary fiber mixture in pediatric patients with controlled chronic constipation. J Pediatr Gastroentero/Nutr. (3): 297-302.

Wu, T., Pan, W., Tang, R., Hwang, S., Wu, L., \& Chen, P. (2011). Constipation in Taiwan elementary school students: a nationwide survey. J Chin Med Assoc. 74 (2): 57-61.

Yang, J., Wang, H., Zhou, L. \& Xu, C. (2012). Effect of dietary fiber on constipation: A meta analysis. World J Gastroenterol. 18 (48): 7378-7383.

Yang, X., Zhang, M., Zhu, H., Tang, Z., Zhao, D., Li, B. \& Gabriel, A. (2016). Epidemiological study: correlation between diet habits and constipation among elderly in Beijing region. World $J$ Gastroenterol. 22 (39): 8806-8811.

You, J., Park, J.. \& Chang, J. (2010). A case-control study on the dietary taurine intake, nutrient status and life stress of functional constipation patients in Korean male college students. J Biomed Sci. 17 (Suppl 1): S41. 
Anexo 1 - Resumo dos estudos incluídos na revisão no que diz respeito à associação entre o consumo alimentar e a obstipação intestinal

\begin{tabular}{|c|c|c|c|c|c|c|c|}
\hline \multirow[b]{2}{*}{ Autores } & \multirow[b]{2}{*}{ Tipo de Estudo } & \multirow[b]{2}{*}{ Idade } & \multirow[b]{2}{*}{ Amostra } & \multicolumn{2}{|c|}{ Instrumento de recolha de dados do } & \multirow[b]{2}{*}{ Resultados } & \multirow[b]{2}{*}{ Conclusões } \\
\hline & & & & Consumo Alimentar & Obstipação intestinal & & \\
\hline $\begin{array}{l}\text { Kindleysides, Kuhn- } \\
\text { Sherlock, Yip e } \\
\text { Poppitt, } 2015\end{array}$ & $\begin{array}{l}\text { Experimental } \\
\text { randomizado duplo- } \\
\text { cego Em Auckland, na } \\
\text { Nova Zelândia }\end{array}$ & $\begin{array}{l}20-63 \\
\text { anos }\end{array}$ & 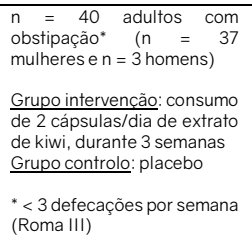 & $\begin{array}{l}\text { A ingestäo de extrato de } \\
\text { kiwi ou o placebo foi } \\
\text { contabilizar da sobra de } \\
\text { cápsulas fornecidas }\end{array}$ & $\begin{array}{l}\text { Número de defecações } \\
\text { semanais (diário fecal) } \\
\text { Consistência das fezes } \\
\text { (escala de Bristol) } \\
\text { Sintomas intestinais } \\
\text { (escala de GSRS) antes e } \\
\text { pós intervenção }\end{array}$ & $\begin{array}{l}\text { Não houve diferenças entre os dois grupos } \\
\text { relativamente ao no de defecações, consistências } \\
\text { das fezes e sintomas intestinais }(p>0,05)\end{array}$ & $\begin{array}{l}\text { Não existe associação entre } \\
\text { o consumo de extrato de kiwi } \\
\text { e a obstipação intestinal. }\end{array}$ \\
\hline $\begin{array}{l}\text { López Román, } \\
\text { Martinez Gonzálvez, } \\
\text { Luque, Pons Miñano, } \\
\text { Vargas Acosta, } \\
\text { Iglesias } \\
\text { Hernández, } 2008\end{array}$ & $\begin{array}{l}\text { Experimental } \\
\text { randomizado duplo- } \\
\text { cego } \\
\text { Em Espanha }\end{array}$ & $\begin{array}{l}\text { Idade } \\
\text { média } \\
47 \text { anos }\end{array}$ & 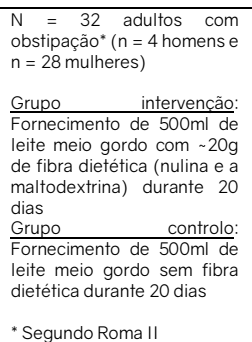 & Sem avaliação & $\begin{array}{l}\text { Diário fecal das } \\
\text { caracteristicas } \\
\text { defecação (critérios de } \\
\text { Roma II), incluindo a } \\
\text { consistência das fezes } \\
\text { (escala de Bristol) } \\
\text { durante } 20 \text { dias }\end{array}$ & $\begin{array}{l}\text { O grupo intervenção apresentou melhoras } \\
\text { significativas relativamente ao menor esforço ao } \\
\text { defecar } \\
(p<0,001) \text {, menos sensação de evacuação } \\
\text { incompleta }(p<0,001) \text {, menos sensação de } \\
\text { obstrução na evacuaçãoo } \\
\text { ( } p<0,001) \text {, menor requência de indivíduos que } \\
\text { defecaram }<3 \text { dias na semana e fezes menos } \\
\text { duras }(p<0,001) \text {. }\end{array}$ & $\begin{array}{l}\text { Associação entre o consumo } \\
\text { de leite com fibra e a a } \\
\text { diminuição da obstipação } \\
\text { intestinal }\end{array}$ \\
\hline $\begin{array}{l}\text { Wu, Pan, Tang, } \\
\text { Hwang, Wu e Chen, } \\
2011\end{array}$ & $\begin{array}{l}\text { Transversal } \\
\text { Em Taiwan }\end{array}$ & $\begin{array}{l}7-12 \\
\text { anos }\end{array}$ & $\begin{array}{l}n=2375 \text { crianças } \\
\text { Meninos } n=1282 \\
\text { Meninas } n=1093 \text { saudáveis }\end{array}$ & $\begin{array}{ll}\text { Questionário } & \text { de } \\
\text { frequência alimentar }\end{array}$ & $\begin{array}{l}\text { Questionário baseado nos } \\
\text { Critério de lowa }\end{array}$ & 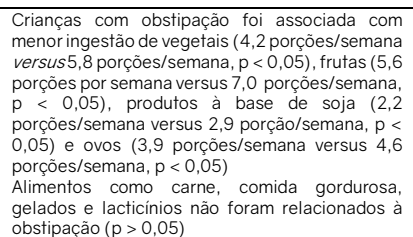 & $\begin{array}{l}\text { Associação entre o consumo } \\
\text { de vegetais, fruta, produtos } \\
\text { de soja e ovos e a diminuição } \\
\text { da obstipação intestinal. } \\
\text { Alimentos como carne, } \\
\text { comida gordurosa, gelados } \\
\text { e lacticinios não têm relação } \\
\text { com obstipação }\end{array}$ \\
\hline $\begin{array}{l}\text { Vitolo, Campagnolo } \\
\text { e Gama, } 2007\end{array}$ & $\begin{array}{l}\text { Transversal } \\
\text { Em São Leopoldo, } \\
\text { Brasil }\end{array}$ & $\begin{array}{l}10-19 \\
\text { anos }\end{array}$ & $\begin{array}{lrr}\mathrm{n}=722 & \text { representam } & \text { os } \\
\text { inquéritos válidos } & \text { e } \\
\text { completos para } & \text { as } \\
\text { conclusōes do estudo. } & \\
\text { meninas } n=479 & \\
\text { Meninos } \mathrm{n}=243\end{array}$ & 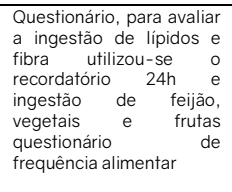 & $\begin{array}{l}\text { Relato da frequência } \leq \\
\text { três vezes na semana e } \\
\text { cuja consistência das } \\
\text { fezes foo considerada } \\
\text { endurecida ouem cibalos, } \\
\text { além do relato de } \\
\text { dificuldade } \\
\text { de evacuar sem uso de } \\
\text { laxantes. }\end{array}$ & $\begin{array}{l}\text { A diminuição de fibra alimentar está associada ao } \\
\text { aumento de obstipação intestinal, tanto para } \\
\text { meninos como meninas }(p<0.05)\end{array}$ & $\begin{array}{l}\text { Associação entre o baixo } \\
\text { consumo de fibra e aumento } \\
\text { da obstipação alimentar. }\end{array}$ \\
\hline
\end{tabular}


EGITAN|A SCIENC।A

$N=25-2019 \quad$ ISSN: $1646-8848$

\begin{tabular}{|c|c|c|c|c|c|c|c|}
\hline Tam, Li e So, 2012 & $\begin{array}{l}\text { Transversal } \\
\text { em Hong Kong }\end{array}$ & $\begin{array}{l}6-15 \\
\text { anos }\end{array}$ & $\begin{array}{l}\mathrm{n}_{1} \quad=2318 \text { crianças, } \\
\text { escollidas aleatoriamente } \\
\text { de três áreas de Hong Kong } \\
\text { de escolas primárias }\end{array}$ & $\begin{array}{l}\text { Questionário } \\
\text { frequência alimentar. }\end{array}$ & $\begin{array}{l}\text { Questionário - QPGS } \\
\text { Rome III }\end{array}$ & $\begin{array}{l}\text { Consumo baixo de fibra }(\mathrm{p}<0,01) \text { e comida fast } \\
\text { food ( } \mathrm{p}<0,01) \text { estão significativamente } \\
\text { associados à obstipação intestinal }\end{array}$ & $\begin{array}{l}\text { Associação estatisticamente } \\
\text { significativa entre a a de obstipação } \\
\text { presença de diminução de } \\
\text { intestinal, a de } \\
\text { fibra alimentar e o aumento } \\
\text { de comida fastfood. }\end{array}$ \\
\hline $\begin{array}{l}\text { Irastorza, Ibañez, } \\
\text { Delgado- } \\
\text { Sanzonetti, Maruri e } \\
\text { Vitoria, } 2010\end{array}$ & $\begin{array}{l}\text { Experimental, } \\
\text { longitudinal não cego } \\
\text { Em Espanha }\end{array}$ & $\begin{array}{l}\text { Entre 6 } \\
\text { meses } \\
\text { e } 14 \\
\text { anos }\end{array}$ & $\begin{array}{l}n=69 \text { crianças } \\
(n=25 \text { meninos e } n=44 \\
\text { meninas) com obstipação** } \\
\text { Grupo de intervençâo: } n=69 \\
\text { crianças } \\
\text { "Segundo Roma III }\end{array}$ & $\begin{array}{l}\text { Registo alimentar no } \\
\text { caderno na última semana } \\
\text { de cada fase. Fase I, dieta } \\
\text { com leite de vaca + fibra } \\
\text { (fruta, vegetais e cereais), } \\
\text { fase II, dieta sem leite de } \\
\text { vaca +fibra (fruta, cereais } \\
\text { e vegetais) } \\
\text { Fase III com leite de vaca } \\
\text { e a fase IV sem leite }\end{array}$ & $\begin{array}{l}\text { Diário no caderno, da } \\
\text { última semana de cada } \\
\text { fase, da frequência de } \\
\text { deposição e consistência } \\
\text { nas quatro fases, usando } \\
\text { a escala de Bristol para } \\
\text { avaliar a consistência }\end{array}$ & $\begin{array}{l}\text { Os movimentos intestinais por semana entre } \\
\text { crianças sem leite de vaca aumentaram } \\
\text { significativamente em comparaçâo com as } \\
\text { crianças com leite de vaca }(p<0.001)\end{array}$ & $\begin{array}{l}\text { Associação estatisticamente } \\
\text { significativa entre o } \\
\text { consumo de leite de vaca e a } \\
\text { presença de obstipação } \\
\text { intestinal crónica, naá tendo } \\
\text { a fibra um papel importante } \\
\text { neste estudo. }\end{array}$ \\
\hline $\begin{array}{l}\text { Asakura, Masayasu } \\
\text { e Sasaki, } 2017\end{array}$ & Transversal, no Japão & $\begin{array}{l}\text { Idade } \\
\text { entre os } \\
5 \text { e } 6 \\
\text { anos }\end{array}$ & $\begin{array}{l}n=5309 \text { crianças } \\
(n=280 \text { meninos e } n=2504 \\
\text { meninas }) \text { de } 380 \text { creches no } \\
\text { Japão }\end{array}$ & $\begin{array}{l}\text { Questionário BDHQ3y, da } \\
\text { frequência alimentar }\end{array}$ & $\begin{array}{l}\text { Questionário baseado }<3 \\
\text { vezes de defecação por } \\
\text { semana, baseado em } \\
\text { Romalll e outros estudos, }\end{array}$ & 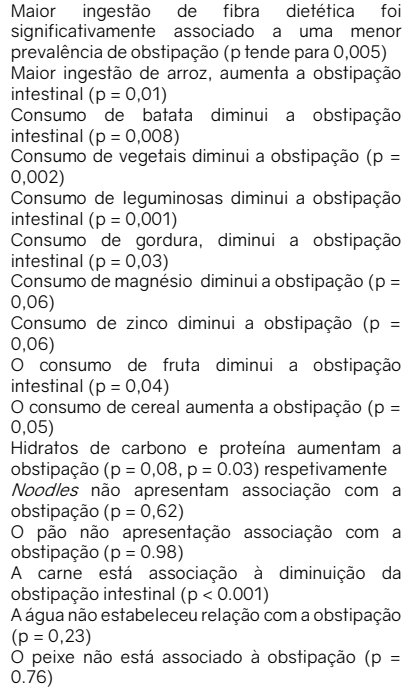 & $\begin{array}{l}\text { Associação estatisticamente } \\
\text { significativa entre um maior } \\
\text { consumo de fruta, vegetais, } \\
\text { carne, leguminosas, batata, } \\
\text { e diminuiçãa de obstipação } \\
\text { Já o consumo de fibra, } \\
\text { gordura, magnésio, zinco e } \\
\text { diminuem a obstipação. } \\
\text { Consumo de hidratos de } \\
\text { carbono aumentam a } \\
\text { obstipação. } \\
\text { Arroz, leva ao aumento de } \\
\text { obstipação intestinal. } \\
\text { Os noodles, peixe, pão e a } \\
\text { água não apresentam } \\
\text { qualquer relaçâo com a } \\
\text { obstipação. }\end{array}$ \\
\hline
\end{tabular}




\begin{tabular}{|c|c|c|c|c|c|c|c|}
\hline $\begin{array}{l}\text { Salehi, Dehghanie } \\
\text { Imanieh, } 2010\end{array}$ & Experimental, no Irão & $\begin{array}{l}1 \quad-20 \\
\text { anos }\end{array}$ & $\begin{array}{l}\mathrm{n}=60 \text { crianças/jovens } \\
(\mathrm{n}=28 \text { meninos e } \mathrm{n}=32 \\
\text { meninas) } \\
\text { com obstipação intestinal } \\
\text { Grupo intervenção: } \mathrm{n}=30 \\
\text { foram sujeitos à alteração à } \\
\text { alimentação como forma de } \\
\text { tratar a obstipaçẫo, } \\
\text { Grupo controlo } n=30 \\
\text { tratamento através de } \\
\text { fármaco (Polietilenoglicol } \\
0.6 \mathrm{~g} / \mathrm{kg} \text { ) }\end{array}$ & $\begin{array}{l}\text { Alteração dos hábitos } \\
\text { alimentares para uma } \\
\text { alimentação rica em fibra, } \\
\text { com diminuiçáo de leite, } \\
\text { iogurte, sumos de fruta, } \\
\text { bebidas e iogurte e } \\
\text { aumento de fruta, } \\
\text { vegetais e liquidos no } \\
\text { grupo intervenção, onde } \\
\text { não é referido o tipo de } \\
\text { IRD utilizado }\end{array}$ & $\begin{array}{l}\text { Avaliação da consistência } \\
\text { (muito firme, firme, } \\
\text { normal e macias) do } \\
\text { grupo intervenção e e o } \\
\text { número de defecaçôes do } \\
\text { grupo caso (2 ou menos } \\
\text { vezes por semana, de } 3 \text { a } \\
6 \text { vezes por semana e } 1 \\
\text { vez por dia) antese apóso } \\
\text { tratamento alimentar do } \\
\text { grupo controlo, sem IRD } \\
\text { definido. }\end{array}$ & $\begin{array}{l}\text { O leite diminuiu na dieta }(p=0.03) \text {, o iogurte }(p= \\
0.041) \text {, a áqua aumentou }(p=0.000) \text {, diminuição } \\
\text { de sumo de fruta }(p=0.057) \text { e outras bebidas }(p \\
=0.006) \\
\text { No grupo intervenção aumento da defecação }(p< \\
0,027) \text {, e a consistência das fezes passou de } \\
\text { muito firme para normal e suave após o treino }(p= \\
0,001) \text {. Também diminuiu no grupo de controlo } \\
(0,047) \text {. } \\
\text { A gravidade da dor e tenesmo durante a } \\
\text { defecação diminuiu devido a dieta equilibrada e } \\
\text { rica em fibra e ingestão de liquidos adequados ( } p \\
=0,012) \text {. }\end{array}$ & $\begin{array}{l}\text { Associação estatisticamente } \\
\text { significativa entre o } \\
\text { consumo de frutae legumes, } \\
\text { ingestão de liquidos (água), } \\
\text { diminui a obstipação } \\
\text { intestinal. } \\
\text { Associaçãa estatisticamente } \\
\text { significativa entre o baixo } \\
\text { consumo de leite, iogurtes, } \\
\text { sumo de fruta e diminuição } \\
\text { da obstipação intestinal no } \\
\text { grupo intervenção. }\end{array}$ \\
\hline $\begin{array}{l}\text { Turan, Dedeli, Bor e } \\
\text { ilter, } 2014\end{array}$ & $\begin{array}{l}\text { Experimental, na } \\
\text { Turquia }\end{array}$ & $\begin{array}{l}27-78 \\
\text { anos }\end{array}$ & $\begin{array}{l}n=20 \text { pacientes } \\
\text { ( } n=13 \text { mulheres e } n=7, \\
\text { descritos com obstipação } \\
\text { intestina* } \\
\text { Grupo de intervenção: } n=10 \\
\text { com trânsito intestinal } \\
\text { normal } \\
\text { Grupo de controlo: } n=10 \\
\text { com transito intestinal lento } \\
\text { "Segundo Roma II }\end{array}$ & $\begin{array}{l}\text { Não existe instrumento de } \\
\text { recolha de dados. } \\
\text { Semanalmente foi dado } \\
\text { Kefir para o tempo de } \\
\text { tratamento durante } 4 \\
\text { semanas } 500 \mathrm{ml} / \text { dia }\end{array}$ & $\begin{array}{l}\text { Diário fecal para avaliara } \\
\text { frequência das fezes, o } \\
\text { grau de tensão e } \\
\text { consistência das fezes a } \\
\text { partir de BSS e consumo } \\
\text { de laxantes. } \\
\text { A satisfaçẫo intestinal foi } \\
\text { avaliada numa escala } \\
\text { analógica visual }(0-10) \\
\text { antes e após ingerir Kefir }\end{array}$ & $\begin{array}{l}\text { Houve uma melhora significativa na satisfação } \\
\text { intestinal após o término do regime de kefir }(p= \\
0,001) \\
\text { No grupo obstipação }(n=10) \text {, diminuiu após a } \\
\text { administração de kefir de } 4 \text { semanas }(p=0,013) \\
\text { No fim da intervenção de kefir de } 4 \text { semanas, } 50 \% \\
\text { destes doentes }(n=6) \text { tinham deixado os } \\
\text { laxantes }(p=0,031) \\
50 \% \text { dos pacientes com obstipação relataram } \\
\text { fezes normais no final do estudo }(p=0,014)\end{array}$ & $\begin{array}{l}\text { Associação estatisticamente } \\
\text { significativa entre o } \\
\text { consumo Suplemento Kefir } \\
\text { (leite fermentado } \\
\text { probiótico) e diminuição da } \\
\text { obstipaçâa intestinal do do } \\
\text { grupo com obstipação } \\
\text { intestinal. }\end{array}$ \\
\hline $\begin{array}{l}\text { You, Park e Chang, } \\
2010\end{array}$ & $\begin{array}{l}\text { Caso controlo } \\
\text { na Coreia }\end{array}$ & $\begin{array}{l}\text { Média } \\
\text { de } 23 \\
\text { anos }\end{array}$ & $\begin{array}{l}N=104 \text { estudantes do sexo } \\
\text { masculino } \\
\text { Grupo de controlo: } n=52 \\
\text { semo obstipacãa intestinal } \\
\text { Grupo de intervenção: } n=52 \\
\text { com obstipaçâã intestinal* } \\
\text { - Segundo Roma II }\end{array}$ & $\begin{array}{l}\text { Recordatório alimentar de } \\
3 \text { dias, para verificar o } \\
\text { consumo de taurina } \\
\text { presente nos alimentos e } \\
\text { outros nutrientes }\end{array}$ & $\begin{array}{l}\text { Questionário segundo os } \\
\text { critérios Roma II }\end{array}$ & 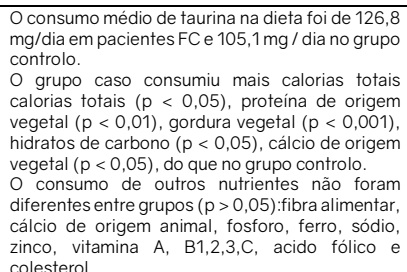 & $\begin{array}{l}\text { Os indivíduos com ingestão } \\
\text { de calorias totais, proteina } \\
\text { vegetal, gordura vegetal, } \\
\text { hidratos de carbono, e cálcio } \\
\text { vegetal de pacientes FC } \\
\text { foram significativamente } \\
\text { maiores em comparação } \\
\text { como o grupo controlo. } \\
\text { Não foi estabelecida } \\
\text { associação da taurina com a } \\
\text { obstipaçâa. }\end{array}$ \\
\hline $\begin{array}{l}\text { Murakami, Okubo, } \\
\text { Takahashi, Hosoi e } \\
\text { Itabashi, 2007 }\end{array}$ & Transversal, no Japão & $\begin{array}{l}18-20 \\
\text { anos }\end{array}$ & $\mathrm{N}_{\text {total }}=3835$ mulheres & $\begin{array}{l}\text { Questionário da história } \\
\text { alimentar feito pelas } \\
\text { mulheres para verificar o } \\
\text { consumo de magnésio, } \\
\text { água e fibra alimentar }\end{array}$ & $\begin{array}{l}\text { Questionário segundo os } \\
\text { critérios de Romal }\end{array}$ & $\begin{array}{l}\text { A baiiea ingestão de água dos alimentos tem } \\
\text { tendencia significativa de }(p>0,04) \text { para } \\
\text { aumentar a obstipação. } \\
\text { A baixa ingestão de magnésio não tem relação } \\
\text { significativa }(p>0,09) \text { com a obstipação, nem a } \\
\text { fibra alimentar. }(p=0.66)\end{array}$ & $\begin{array}{l}\text { A baixa ingestão da água nos } \\
\text { alimentos aumenta a a } \\
\text { obstipação intestinal. } \\
\text { O magnésio e a fibra } \\
\text { alimentar naõo têm relação } \\
\text { com a obstipaçâao intestinal. }\end{array}$ \\
\hline $\begin{array}{l}\text { Lopes e Victoria, } \\
2008\end{array}$ & $\begin{array}{l}\text { Caso controlo } \\
\text { Em São Paulo, Brasil }\end{array}$ & $\begin{array}{l}\text { Média } \\
\text { de } 45 \\
\text { anos }\end{array}$ & $\begin{array}{l}n=48 \text { pessoas } \\
\text { Grupo de intervenção: } n=30 \\
(n=26 \text { mulheres } n=4 \\
\text { homens) com obstipação } \\
\text { intestinal }\end{array}$ & $\begin{array}{ll}\text { Registo alimentar } & \text { da } \\
\text { ingesta da fibra alimentar } \\
\text { durante } 7 & \text { dias } \\
\text { consecutivos } & \end{array}$ & $\begin{array}{l}\text { Questionário para avaliar } \\
\text { a intensidade da } \\
\text { obstipação a partir da } \\
\text { escala de AGACHAN e o } \\
\text { tempo de trânsito } \\
\text { colónico, total e }\end{array}$ & $\begin{array}{l}\text { O grupo caso consumiu mais fibra que o grupo } \\
\text { controlo. ( } p<0,001) \\
\text { Não houve correlação entre a ingestão de fibra e a } \\
\text { intensidade da obstipação no grupo caso. ( } p= \\
0,913)\end{array}$ & $\begin{array}{l}\text { Não houve relação entre a } \\
\text { ingestão de fibras, o } \Pi C \\
\text { total e segmentar e a } \\
\text { intensidade de obstipação } \\
\text { no grupo caso, pelo que a } \\
\text { intensidade da obstipação }\end{array}$ \\
\hline
\end{tabular}




\begin{tabular}{|c|c|c|c|c|c|c|c|}
\hline & & & $\begin{array}{l}\text { Grupo de controlo: } n=18 \\
\text { pessoas }(n=3 \text { homenseen } n= \\
15 \text { mulheres), sem queixas } \\
\text { digestivas, sem obstipaçẫo } \\
\text { intestinal } \\
\text { - Segundo Roma II }\end{array}$ & & $\begin{array}{l}\text { segmentar a partir da } \\
\text { técnica dos marcadores } \\
\text { radiopacos }\end{array}$ & $\begin{array}{l}\text { O TTC total e segmentar (direito e sigmóide) foi } \\
\text { maior no grupo controlo do que no caso. ( } p< \\
0,005, p<0,05 \text { e } p<0,05 \text {, respetivamente) }\end{array}$ & $\begin{array}{l}\text { não depende apenas da } \\
\text { ingestão de fibras. }\end{array}$ \\
\hline $\begin{array}{l}\text { Chien, Liou e Chang, } \\
2011\end{array}$ & $\begin{array}{l}\text { Transversal } \\
\text { em Taiwan }\end{array}$ & $\begin{array}{l}10-18 \\
\text { anos }\end{array}$ & $\begin{array}{l}N=14626 \\
(n=6713 \text { meninas en }=7913 \\
\text { meninos })\end{array}$ & $\begin{array}{l}\text { Recordatório do consumo } \\
\text { médio dos alimentos nos } \\
\text { últimos } 7 \text { dias }\end{array}$ & $\begin{array}{lr}\text { Através de } & \text { um } \\
\text { questionário, com uma } \\
\text { questão acerca da } \\
\text { frequência de defecação } \\
\text { (< menos de três vezes } \\
\text { por semana tem } \\
\text { obstipação) }\end{array}$ & $\begin{array}{l}\text { Aumento de líquidos é estatisticamente } \\
\text { significativo na diminuição da obstipação } \\
\text { intestinal }(p<0,001) \\
\text { Aumento de frutas e vegetais é estatisticamente } \\
\text { significativo na diminuição da obstipação } \\
\text { intestinal }(p<0,001) \\
\text { Quanto mais alimentos básicos se comer com } \\
\text { grãos inteiros menoré a obstipação intestinal ( } p< \\
0,001 \text { ) }\end{array}$ & $\begin{array}{l}\text { A associação é } \\
\text { estatisticamente } \\
\text { significativa entre o aumento } \\
\text { da ingestão de liquidos, } \\
\text { vegetais, frutas e grãos } \\
\text { integrais entre os os } \\
\text { adolescentes com niveis de } \\
\text { ingestão muito baixos pode } \\
\text { prevenir e controlar a } \\
\text { obstipação intestinal }\end{array}$ \\
\hline $\begin{array}{l}\text { Weber, Toporovski, } \\
\text { Tahan, Neufeld e de } \\
\text { Morais, } 2014\end{array}$ & $\begin{array}{l}\text { Experimental, } \\
\text { randomizado duplo } \\
\text { cego. } \\
\text { SãoPaulo, Brasil }\end{array}$ & $\begin{array}{l}4-12 \\
\text { anos }\end{array}$ & 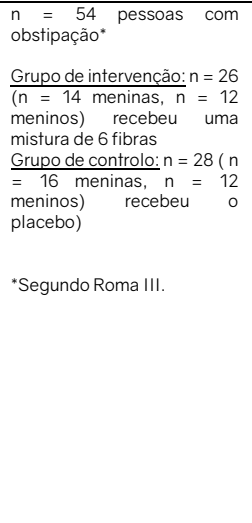 & $\begin{array}{l}\text { Adose de fibra foidefinida } \\
\text { de acordo ao peso } \\
\text { corporal: } 3,8 \mathrm{~g} \text { de uma } \\
\text { mistura de fibras ou } \\
\text { placebo (1 colher) diluido } \\
\text { em } 200 \text { ml de uma bebida } \\
\text { de leite com chocolate a } \\
\text { crianças com peso até } 18 \\
\mathrm{~kg} \text { e } 7,6 \mathrm{~g} \text { de fibra ou } \\
\text { placebo } \\
\text { (2 colheres de sopa) foi } \\
\text { dada na mesma forma } \\
\text { para crianças com peso > } \\
18 \mathrm{~kg} \text {. verificação do } \\
\text { cumprimento da terapia } \\
\text { cumprime } \\
\text { foi verificado a partir do } \\
\text { peso das latas de produto } \\
\text { entregues aos } \\
\text { investigadores. } \\
\text { Também foi avaliada a } \\
\text { palatabilidade ou seja o } \\
\text { gosto pelo alimento } \\
\text { consumido (mau, razoável } \\
\text { e bom) (m) }\end{array}$ & $\begin{array}{l}\text { 1 e } 3 \text { semana foi feita } \\
\text { recolha de informação a } \\
\text { partit do telefone. } 2 \text { e } 4 \\
\text { semana recolha a partir da } \\
\text { visita ao hospital. } \\
\text { Quem fornece o } \\
\text { tratamento em casa teve } \\
\text { que preencher dois } \\
\text { questionários: } \\
\text { loQuestionário sobre a } \\
\text { frequência diária de } \\
\text { defecação e o tipo de } \\
\text { fezes eliminadas com } \\
\text { base na Escala Bristol } \\
\text { Stool Form } \\
\text { 20.Questionário sobre os } \\
\text { sintomas relacionados à } \\
\text { obstipação intestinal eao } \\
\text { uso de suplementação. } \\
\text { Foi avaliado também os } \\
\text { CTT a partir da ténica do } \\
\text { marcadores radiopaticos }\end{array}$ & $\begin{array}{l}\text { Segundo os resultados obtidos, avaliação } \\
\text { primária, falha terapêutica, foi idêntica entre os } \\
\text { grupo caso e o controlo ( } p<0,093) \\
\text { Já em relação ao desfecho secundário, foi obtido } \\
\text { só de quem cumprius otratamento } N=44 \text { pessoas, } \\
\text { onde o aumento médio nos movimentos } \\
\text { intestinais diários foi de } 0,53 \text { no grupo da mistura } \\
\text { de fibras alimentares e } 0,23 \text { no grupo de controlo } \\
\text { ( } p=0,014) \text {. Os pacientes do grupo de mistura de } \\
\text { fibras alimentares }(60,0 \%) \text { passaram fezes não } \\
\text { endurecidas mais frequentemente do que no } \\
\text { grupo controle ( } 16,7 \%, p=0,003) \text {, portanto é } \\
\text { estatisticamente significativas as diferenças } \\
\text { obtidas entre os grupos. } \\
O \text { nivel palatabilidade foi estatisticamente } \\
\text { significativa, melhor no grupo com fibra } \\
\text { adicionada em fez de maltodextrina ( } p=0,007) \text {. } \\
\text { Os CTT foram semelhantes para ambos os grupos } \\
\text { no entanto a amostra era de } N=43 \text { com } \\
\text { desistenceia de mais uma pessoa. } \\
O \text { consumo de fibra antes do estudo foi idêntico } \\
\text { entre os grupos. ( }(p=0,993) \text {. }\end{array}$ & $\begin{array}{l}\text { A mistura de fibras não } \\
\text { impediu a suspensão de } \\
\text { amaciadores de fezes nem } \\
\text { levou à redução dos CTT, } \\
\text { contudo, existe associação } \\
\text { estatisticamente } \\
\text { significativa da mistura de } \\
\text { fibra promover uma maior } \\
\text { frequência de defecação e } \\
\text { uma melhoria na na } \\
\text { consistência das fezes. }\end{array}$ \\
\hline $\begin{array}{l}\text { Dehghani, } \\
\text { Ahmadpour, } \\
\text { Haghighat, Kashef, } \\
\text { Imanieh e Soleimani, } \\
2012\end{array}$ & $\begin{array}{l}\text { Experimental, } \\
\text { randomizado, no Irão }\end{array}$ & $\begin{array}{l}1-13 \\
\text { anos }\end{array}$ & $\begin{array}{l}\mathrm{n}=140 \text { pessoas }(\mathrm{n}=73 \\
\text { meninos e } \mathrm{n}=67 \text { meninas }) \\
\text { com obstipação* } \\
\text { Grupo de intervenção: } n=70 \\
(\mathrm{n}=39 \text { meninos } \mathrm{e} n=31 \\
\text { meninas dieta s/leite de } \\
\text { vaca durante } 4 \text { semanas }+2 \\
\text { com leite de vaca } \\
\text { Grupo de controlo: } n=70(\mathrm{n}) \\
34 \text { meninos } \mathrm{e} n=36 \\
\text { meninas) dieta com leite de } \\
\text { vaca durante } 4 \text { semanas }\end{array}$ & $\begin{array}{l}\text { Diário alimentar do tipo e } \\
\text { quantidade de leite } \\
\text { ingerido }\end{array}$ & $\begin{array}{l}\text { Diário sobre o número de } \\
\text { evacuaçôes e dificuldade } \\
\text { da criança em defecar } \\
\text { posteriormente avaliados } \\
\text { a partir dos critérios de } \\
\text { Roma III }\end{array}$ & $\begin{array}{l}\text { Após } 4 \text { semanas, o grupo intervenção } \\
\text { responderam que os sintomas diminuiram em } \\
\text { comparação ao grupo controlo }(p=0,0001) \text {. } \\
\text { Contudo nas } 2 \text { semanas que adicionaram leite a } \\
\text { dieta } 24 \text { dos } 56 \quad(42,8 \% \text { afirmaram } \\
\text { desenvolveram novamente obstipação. }\end{array}$ & $\begin{array}{l}\text { Associaçãa estatisticamente } \\
\text { significativa entre o } \\
\text { consumo leite de vaca e } \\
\text { aumento da obstipação } \\
\text { intestinal, podendo ser } \\
\text { associada a alergia ao leite } \\
\text { de vaca. }\end{array}$ \\
\hline
\end{tabular}




\begin{tabular}{|c|c|c|c|c|c|c|c|}
\hline & & & *Segundo Roma III & & & & \\
\hline $\begin{array}{l}\text { Inan, Aydiner, Tokuc, } \\
\text { Aksu, Ayvaz, Ayhan } \\
\text { etal., 2007 }\end{array}$ & $\begin{array}{l}\text { Transversal } \\
\text { na Turquia }\end{array}$ & $\begin{array}{l}7-12 \\
\text { anos }\end{array}$ & $\begin{array}{l}n=1689 \text { crianças }(n=864 \\
\text { mulheres e } n=825 \text { homens) }\end{array}$ & $\begin{array}{l}\text { Questionário frequência } \\
\text { alimentar }\end{array}$ & $\begin{array}{l}\text { Questionário com critérios } \\
\text { de NASPGHAN para } \\
\text { avaliar a obstipação }\end{array}$ & $\begin{array}{l}\text { Crianças constipadas foram relatados para ter um } \\
\text { maior consumo de leite, queijo, iogurte, biscoitos, } \\
\text { pão e macarrão e um menor consumo de frutas e } \\
\text { vegetais }(p<0,05 \text {. Nâo havia diferença } \\
\text { estatisticamente significativa entre o consumo de } \\
\text { nozes e arroz }(p>0,05)\end{array}$ & $\begin{array}{l}\text { Associação estatisticamente } \\
\text { significativa entre o aumento } \\
\text { de iogurte, queijo, massa, } \\
\text { pão e bolachas e um menor } \\
\text { consumo de horticolas e } \\
\text { fruta no aumento da } \\
\text { obstipação intestinal. } \\
\text { Não há relação entre o arroz } \\
\text { e nozes a obstipação } \\
\text { intestinal. }\end{array}$ \\
\hline $\begin{array}{l}\text { Maffei e Vicentini, } \\
2011\end{array}$ & $\begin{array}{l}\text { Prospetivo } \\
\text { longitudinal, em São } \\
\text { Paulo, Brasil }\end{array}$ & $\begin{array}{l}\text { Média } \\
\text { de } 7 \\
\text { anos }\end{array}$ & $\begin{array}{l}n \quad=28 \text { crianças } \quad \text { com } \\
\text { obstipação crónica intestinal } \\
\text { Total de visitas }=108\end{array}$ & $\begin{array}{l}\text { Questionário de } \\
\text { frequência alimentar dos } \\
\text { hábitos das } 4 \text { semanas } \\
\text { anteriores aplicado em } \\
\text { cada visita }\end{array}$ & $\begin{array}{l}\text { Questionário segundo } \\
\text { critério de Boston das } 4 \\
\text { semanas anteriores } \\
\text { aplicado em cada visita }\end{array}$ & $\begin{array}{l}\text { Maior ingestão de fibra alimentar em crianças que } \\
\text { tiveram melhorias e ausência de complicaçôes } \\
\text { nas visitas }(p=0,05) \\
\text { Na visita } 1 \text { houve mais complicações intestinais ( } p \\
=0,05) \\
\text { Entre a V2 e V5 houve mais melhorias que } \\
\text { complicações }(p=0,03)\end{array}$ & $\begin{array}{l}\text { Associação estatisticamente } \\
\text { significativa entre o } \\
\text { consumo de farelo trigo } \\
\text { (fibra alimentar) e } \\
\text { diminuição de obstipação } \\
\text { intestinal. }\end{array}$ \\
\hline $\begin{array}{ll}\text { Machado } & \text { e } \\
\text { Capelarim, 2010 } & \end{array}$ & $\begin{array}{l}\text { Experimental } \\
\text { Iongitudinal, no Brasil }\end{array}$ & $\begin{array}{l}18-74 \\
\text { anos, } \\
\text { com } \\
\text { uma } \\
\text { média } \\
\text { de } 46 \\
\text { anos }\end{array}$ & $\begin{array}{l}n=50 \text { pessoas }(n=46 \\
\text { mulheres e } n=4 \text { homens }) \\
\text { com obstipação* }\end{array}$ & $\begin{array}{l}\text { Foi dado na 10abordagem } \\
\text { oferta de fibra em doses } \\
\text { diárias (4 g/dia, } \\
\text { aumentando } \\
\text { quinzenalmente) } \\
\text { 20 Abordagem } \\
\text { Questionário sobre a } \\
\text { adesão à fibra alimentar } \\
\text { após 4 meses combase na } \\
\text { quantidade estipulada }\end{array}$ & 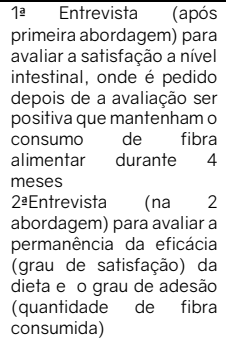 & $\begin{array}{l}\text { Foi encontrada associação estatisticamente } \\
\text { significante entre adesão e eficácia } \\
(p=0,008) \text {. }\end{array}$ & $\begin{array}{l}\text { Associaçãa estatisticamente } \\
\text { significativa entre o } \\
\text { consumo de fibra alimentare } \\
\text { a diminiuição de obstipação } \\
\text { intestinal, embora a adesão } \\
\text { seja baixa ao consumo de } \\
\text { fibra alimentar. }\end{array}$ \\
\hline $\begin{array}{l}\text { Yang, Zhang, Zhu, } \\
\text { Tang, Zhao, Li e } \\
\text { Gabriel, } 2016\end{array}$ & Transversal, na China & $\begin{array}{l}> \\
\text { anos }\end{array}$ & $\begin{array}{l}n=2776 \text { pessoas } n=1213 \\
\text { homens e } n=1563 \text { mulheres }\end{array}$ & $\begin{array}{ll}\text { Questionário } & \text { de } \\
\text { frequência alimentar }\end{array}$ & $\begin{array}{l}\text { Questionário baseado na } \\
\text { China Obstipação Crónica } \\
\text { Diagnóstico Tratamento } \\
\text { Orientação (2013) }\end{array}$ & $\begin{array}{l}\text { Diferença estatisticamente significativa entre } 0 \\
\text { consumo de alimentos básicos e a diminuição da } \\
\text { obstipação }(p<0,05) \\
\text { Não houve diferença estatisticamente } \\
\text { significativa entre o consumo de ovos e a } \\
\text { obstipação }(p<0,05) \\
\text { Houve diferença estatisticamente significativa } \\
\text { entre o consumo de peixe e a diminuição de } \\
\text { obstipaçâo }(p<0,01) \\
\text { Diferença estatisticamente significativa entre o } \\
\text { consumo de vegetais e fruta e a diminuição de } \\
\text { obstipação }(p<0,05)\end{array}$ & $\begin{array}{l}\text { Associação estatisticamente } \\
\text { significativa entre o } \\
\text { consumo de fibra alimentar a } \\
\text { partir de legumes, fruta, } \\
\text { alimentos básico e a a } \\
\text { diminuição da obstipação } \\
\text { intestinal. }\end{array}$ \\
\hline Ayaz e Hisar, 2014 & $\begin{array}{l}\text { Prospetivo } \\
\text { Longitudinal na Turquia } \\
\text { com programa } \\
\text { educacional para o } \\
\text { consumo de (consumir } \\
\text { früa, vegetais, água, } \\
\text { grãos e evitar álcool, } \\
\text { chá e café devem ser }\end{array}$ & $\begin{array}{l}\geq \text { a } 18 \\
\text { anos }\end{array}$ & $\begin{array}{l}n=37 \text { mulheres com } \\
\text { obstipaçâo intestinal, } \\
\text { segundo CSI, CVAS e BSC }\end{array}$ & $\begin{array}{l}\text { Questionário frequência } \\
\text { alimentar } \\
\text { autoadministrado com um } \\
\text { tópico sobre o consumo } \\
\text { de frutas, vegetais e água }\end{array}$ & $\begin{array}{l}\text { Questionário com CSI, } \\
\text { CVAS e BSC }\end{array}$ & $\begin{array}{l}82,9 \% \text { das mulheres relataram consumir entre } \\
\text { uma e três porções de vegetais ou frutas por dia, } \\
85,5 \% \text { bebiam menos de oito copos de água por } \\
\text { dia e } 51,4 \% \text { relataram não consumir sumo de fruta } \\
\text { por dia e } 66,7 \% \text { afirmaram beber entre } 1 \text { a } 3 \text { copos } \\
\text { de chá e café. } \\
\text { As escalas médias totais de CSI e CVAS foram } \\
\text { significativamente diminuidos após o programa }\end{array}$ & $\begin{array}{l}\text { Associação estatisticamente } \\
\text { significativa entre o } \\
\text { programa educacional e a } \\
\text { diminuição de obstipação } \\
\text { intestinal. } \\
\text { Uma vez que o programa } \\
\text { educacional envolve } \\
\text { alimentação, aconselhando } \\
\end{array}$ \\
\hline
\end{tabular}




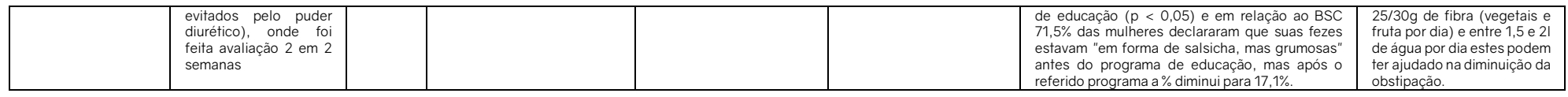

